\title{
Sparcl1 promotes nonalcoholic steatohepatitis progression in mice through upregulation of CCL2
}

\author{
Bin Liu, ${ }^{1,2}$ Liping Xiang, ${ }^{1}$ Jing Ji, ${ }^{2}$ Wei Liu, ${ }^{2}$ Ying Chen, ${ }^{1}$ Mingfeng Xia, ${ }^{1}$ Yuejun Liu, ${ }^{1}$ Wenyue Liu, ${ }^{3}$ Peiwu Zhu, ${ }^{4}$ Yi Jin, ${ }^{5}$ Yu Han, ${ }^{6}$ \\ Jieli Lu, ${ }^{7}$ Xiaoying Li, ${ }^{1}$ Minghua Zheng, ${ }^{8,9}$ and Yan Lu' \\ 'Department of Endocrinology and Metabolism, Fudan Institute for Metabolic Diseases, Zhongshan Hospital, Fudan University, Shanghai, China. ${ }^{2}$ liangsu Key Laboratory of Marine Pharmaceutical \\ Compound Screening, College of Pharmacy, Jiangsu Ocean University, Lianyungang, China. ${ }^{3}$ Department of Endocrinology, ${ }^{4}$ Department of Clinical Laboratory, ${ }^{5}$ Department of Pathology, ${ }^{6}$ Department of \\ Gastrointestinal Surgery, The First Affiliated Hospital of Wenzhou Medical University, Wenzhou, China. ${ }^{7}$ Shanghai National Clinical Research Center for Metabolic Diseases, Shanghai Institute of Endocrine \\ and Metabolic Diseases, Ruijin Hospital, Shanghai Jiao Tong University School of Medicine, Shanghai, China. ${ }^{8}$ MAFLD Research Center, Department of Hepatology, the First Affiliated Hospital of Wenzhou \\ Medical University, Wenzhou, China. ${ }^{9}$ Key Laboratory of Diagnosis and Treatment for The Development of Chronic Liver Disease of Zhejiang Province, Wenzhou, China.
}

\begin{abstract}
Nonalcoholic fatty liver disease (NAFLD) represents a spectrum of chronic liver disease ranging from simple steatosis (NAFL) to nonalcoholic steatohepatitis (NASH). However, the molecular mechanisms of NASH progression remain incompletely understood. White adipose tissue (WAT) has emerged as an important endocrine organ and contributes not only to the initial stage of NAFLD, but also to its severity. In the current study, through transcriptomic analysis we identified increased expression of Sparcl1, a secreted glycoprotein, in the WAT from NASH mice. Plasma Sparcl1 levels were similarly elevated and positively correlated with hepatic pathological features in NASH patients. Functional studies showed that both chronic injection of recombinant Sparcl1 protein and overexpression of Sparcl1 exaggerated hepatic inflammation and liver injury in mice. In contrast, genetic ablation of Sparcl1, knockdown of Sparcl1 in WAT, and treatment with a Sparcl1-neutralizing antibody dramatically alleviated diet-induced NASH pathogenesis. Mechanistically, Sparcl1 promoted the expression of C-C motif chemokine ligand 2 (CCL2) in hepatocytes through binding to Toll-like receptor 4 (TLR4) and activation of the NF-кB/p65 signaling pathway. Genetically or pharmacologically blocking the CCL2/CCR2 pathway attenuated the hepatic inflammatory response evoked by Sparcl1. Thus, our results demonstrated an important role for Sparcl1 in NASH progression, suggesting a potential target for therapeutic intervention.
\end{abstract}

\section{Introduction}

In the past decade, the prevalence of obesity has substantially increased in both the adults and children (1) due to rapid changes in lifestyle. Obesity is associated with high risk for many metabolic disorders, such as type 2 diabetes mellitus, nonalcoholic fatty liver disease (NAFLD), and hyperlipidemia. NAFLD comprises a spectrum of liver conditions ranging from simple steatosis (NAFL) to the more serious nonalcoholic steatohepatitis (NASH) $(2,3)$. Although NAFL is considered benign, $10 \%-20 \%$ of patients can progress to $\mathrm{NASH}$, which has become the most rapidly growing indication for liver transplantation in the United States (4).

A hallmark of NASH is the prominent hepatic inflammatory response $(5,6)$. Animal studies have shown that inflammation in hepatocytes is an important link between the initial metabolic stress and subsequent hepatocyte death, liver injury, and fibrogenesis in NASH (7). In particular, while patients with simple steatosis

\section{Delated Commentary: https://doi.org/10.1172/JCl153640}

Authorship note: BL and LX contributed equally to this work. Conflict of interest: The authors have declared that no conflict of interest exists. Copyright: (5) 2021, American Society for Clinical Investigation.

Submitted: September 30, 2020; Accepted: August 31, 2021; Published: October 15, 2021. Reference information: J Clin Invest. 2021;131(20):e144801.

https://doi.org/10.1172/JCI144801. exhibit a benign clinical course, hepatic inflammation and its main consequence, fibrosis, is a key determinant of long-term adverse outcomes $(8,9)$. Nevertheless, the cellular and molecular mechanisms underlying the activation of hepatic inflammation remain largely unknown. One of the key factors in hepatocytes is the membrane protein Toll-like receptor 4 (TLR4) (10), which functions as a receptor for endotoxins such as lipopolysaccharide (LPS) (11). However, abundance of these endogenous ligands is already elevated at the NAFL stage. LPS levels in plasma and hepatocytes were higher in patients with NAFL or NASH and correlated with $\mathrm{NF}-\kappa \mathrm{B}$ activation, as compared with normal subjects (12). However, no difference was found between patients with NAFL and NASH (12). In addition, another study showed a positive association between LPS-binding protein and hepatic fat deposition, but not with the severity of lobular inflammation or fibrosis (13). These direct and indirect pieces of evidence strongly suggest that molecules or proteins that selectively activate TLR4 signaling during NASH progression remain to be identified.

One of the key risk factors for obesity-associated diseases is attributed to the expansion of white adipose tissue (WAT). Decades of studies have established WAT not only as an inert energy-storage site, but also as a major endocrine organ with metabolic regulatory roles. WAT dynamically participates in the pathogenesis of all aspects of NAFLD through release of fatty acids, proinflammatory cytokines, and multiple polypeptides 
$(14,15)$. These polypeptides are collectively termed adipokines, with multipotent physiological and pathophysiological effects on chronic liver diseases (14-16). Among them, leptin and adiponectin are the best-studied adipokines that are associated with the development and progression of NAFLD (17). It has been reported that plasma leptin levels were higher in NASH patients than those in NAFL patients or controls $(18,19)$, while hypoadiponectinemia correlated with the severity of liver pathology in NASH $(20,21)$. In addition, adipose tissue-expressed neuregulin 4 (Nrg4) can protect hepatocytes from cell death, thereby attenuating diet-induced $\mathrm{NASH}$ in mice (22). As such, identification of novel adipokines in NASH progression may help to reveal its molecular mechanisms and provide a potential therapeutic strategy for treatment.

In the present study, through transcriptomic analysis we found that secreted protein acidic and rich in cysteine-like protein 1 (Sparcl1) is upregulated in the WAT of diet-induced NASH mouse models. More importantly, elevated plasma Sparcl1 levels correlated with severity of NASH in a human cohort. Sparcl1 is a secreted glycoprotein belonging to a large family of matricellular proteins. It is implicated in the regulation of cell proliferation and migration, by which it regulates embryogenesis, tissue remodeling, and tumorigenesis $(23,24)$. This family also includes several other molecules, including Sparc, thrombospondin 1 and 2, osteopontin, and tenascin $\mathrm{C}$ and $\mathrm{X}$, which have been linked to the development of insulin resistance, chronic liver diseases, and diabetic retinopathy and nephropathy (25-28). However, whether Sparcl1 plays a role in obesity-associated metabolic abnormalities has not yet been explored.

\section{Results}

Construction of mouse models for NAFL and NASH. Murine dietary models of NAFLD/NASH widely vary in their effects. Although many models exhibit hepatic lipid accumulation, their histological appearance may not resemble human NASH (3). C57BL/6J mice usually do not develop steatohepatitis or fibrosis on a high-fatdiet (HFD), while mice fed a methionine- and choline-deficient diet lose rather than gain weight and are not insulin resistant (3). Therefore, these mouse models might not be appropriate for the study of obesity-associated NASH. Recent studies described that long-term consumption of HFD additionally containing high fructose and high cholesterol (HFHC diet) can induce obesity, hepatic steatosis, and liver inflammation and injury, which appears to closely resemble human NASH $(22,29)$. Thus, C57BL/6J male mice were fed a normal chow diet or HFHC diet for 2 different periods: 12 weeks and 28 weeks (Supplemental Figure 1A; supplemental material available online with this article; https://doi. org/10.1172/JCI144801DS1). Compared with mice fed a normal chow diet, mice fed an HFHC diet for 12 weeks developed obesity and liver steatosis (Supplemental Figure 1, B-E). However, plasma levels of alanine aminotransferase (ALT) and aspartate aminotransferase (AST), two markers of liver injury, were unaffected (Supplemental Figure 1, F and G). mRNA levels of the majority of genes involved in hepatic inflammation and liver fibrosis were unaltered (Supplemental Figure 1H). In agreement, staining of F4/80, a unique marker of murine macrophages, was comparable between the 2 groups of mice (Supplemental Figure 1I). In addition, we compared HFHC mice with HFD mice at the same age, as described in our previous studies $(30,31)$. We found that body weight, hepatic triglyceride (TG) content, and mRNA expression levels of lipogenic genes were comparable between these 2 obesity models (data not shown). These results suggest that mice fed a 12-week HFHC diet may have similar hepatic metabolic changes to those of HFD mice at the same age.

On the other hand, in addition to the exaggerated body weight, liver/body weight ratio, and hepatic TG retention (Supplemental Figure 2, A-D), mice fed an HFHC diet for 28 weeks exhibited higher plasma ALT and AST levels (Supplemental Figure 2, E and F). Moreover, F4/80 staining and expression of genes related to hepatic inflammation and liver fibrosis were significantly upregulated (Supplemental Figure 2, G and H). These observations indicate that short-term feeding (12 weeks) of HFHC diet may induce simple steatosis (NAFL) in mice, whereas long-term feeding (28 weeks) can cause NASH.

Identification of Sparcl1 as a WAT-secreted protein in NASH mice. WAT, especially visceral adipose tissue (vAT), has been considered a crucial contributor to NAFLD development through multiple mechanisms (32). Classically activated proinflammatory macrophages during vAT expansion may influence systemic insulin sensitivity and hepatic inflammation through delivery of inflammatory mediators. Moreover, white adipocytes are capable of secreting not only fatty acids but also a class of secretory proteins that modulate the homeostasis of distant organs, including the liver $(16,17,33)$. To identify potential WAT-enriched secretory factors involved in NASH progression, we firstly compared the expression profile of epididymal WAT (eWAT) from mouse models of NAFL and NASH mentioned above by RNAsequencing (RNA-Seq) analysis (Supplemental Figure 3A). Then, using a $P$ value of 0.05 and fold change greater than 1.5 as cutoff points, we undertook intersection analysis of the 2 groups of dysregulated genes (differentially expressed genes, DEGs). While 392 genes were dysregulated in both mouse models, a total of 823 and 1420 genes were selectively dysregulated in the eWAT of NAFL and NASH mice, respectively (Figure 1, A and B, and Supplemental Table 1). Functional enrichment analysis showed that many DEGs in NASH mice were enriched in extracellular matrix remodeling (Figure $1 \mathrm{C}$ ), indicating the disorganization of basement membrane components in obese adipose tissues, as described previously (34).

Then, these 1420 DEGs in NASH mice were compared with a previously published mouse cytokine database (GSE10246, NCBI Gene Expression Omnibus), which contains a set of mouse genes predicted to carry an encoded signal peptide for secretion. As a result, 54 cytokines were increased and 64 cytokines were decreased in the eWAT of NASH mice (Figure 1, D and E). We observed downregulation of $\mathrm{Nrg} 4$ (Figure 1E), a newly identified adipokine, which is consistent with the results from a recent study (22). In addition, we observed upregulation of Sparc (Figure 1D). Sparc expression has been shown to be predominant in adipose tissues and correlated with fat mass in humans (35). Animal studies confirmed that abnormal expression of Sparc plays an important role in metabolic disorders and hepatic injury $(25,36)$. The downregulation of $\mathrm{Nrg} 4$ and upregulation of Sparc in the NASH group were further confirmed by quantitative real-time PCR (qRT-PCR) analysis (Supplemental Figure 3B). Interestingly, we 
A

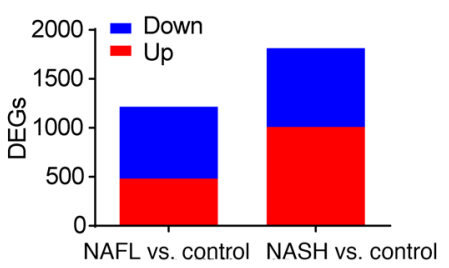

D

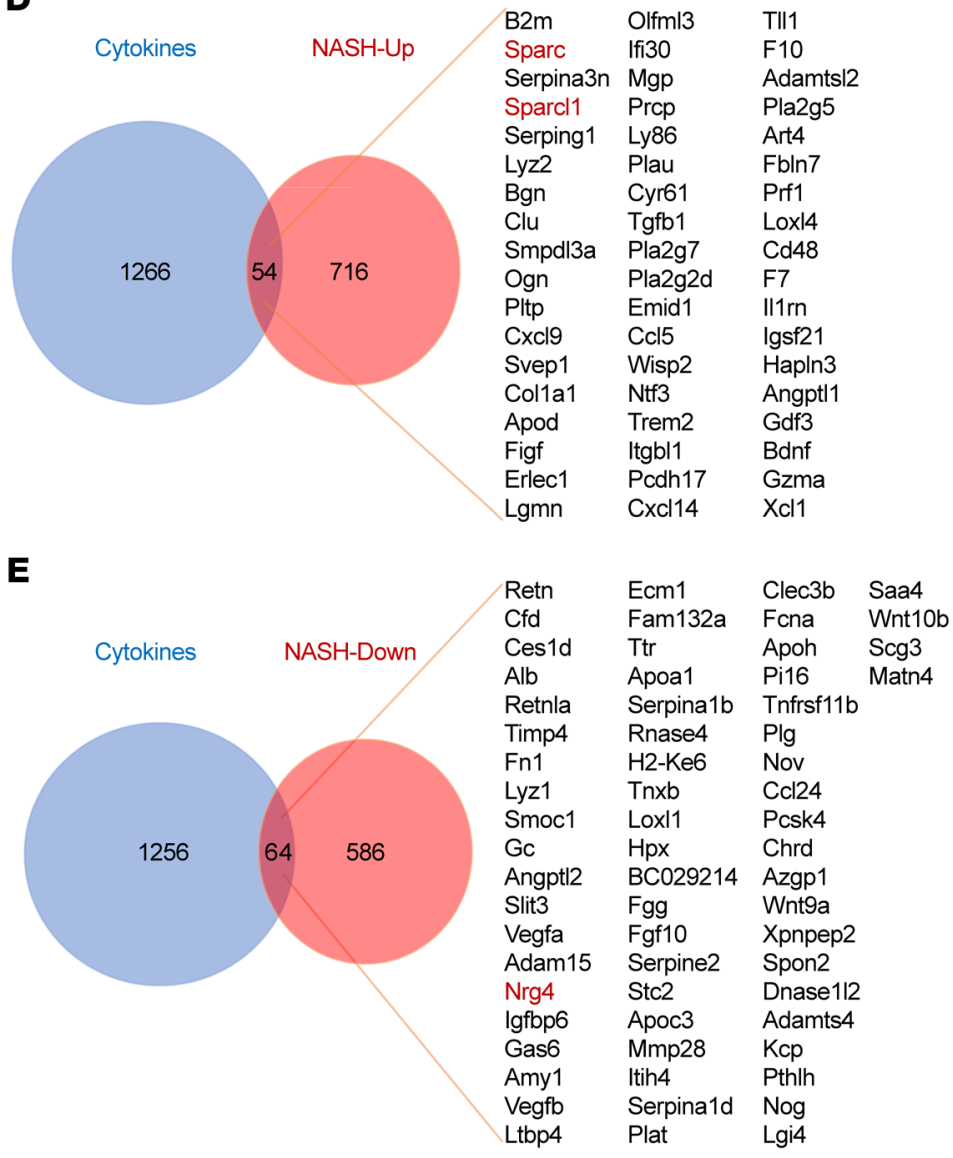

B $\begin{array}{cc}\text { DEGs } & \text { DEGs } \\ \text { (NAFL vs. control) } & \text { (NASH vs. control) }\end{array}$

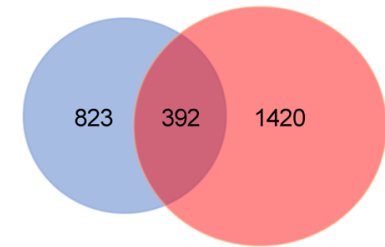

$\begin{array}{lll}\text { B2m } & \text { Olfml3 } & \text { TIl1 } \\ \text { Sparc } & \text { Ifi30 } & \text { F10 } \\ \text { Serpina3n } & \text { Mgp } & \text { Adamts|2 } \\ \text { Sparcl1 } & \text { Prcp } & \text { Pla2g5 } \\ \text { Serping1 } & \text { Ly86 } & \text { Art4 } \\ \text { Lyz2 } & \text { Plau } & \text { Fbln7 } \\ \text { Bgn } & \text { Cyr61 } & \text { Prf1 } \\ \text { Clu } & \text { Tgfb1 } & \text { Loxl4 } \\ \text { Smpdl3a } & \text { Pla2g7 } & \text { Cd48 } \\ \text { Ogn } & \text { Pla2g2d } & \text { F7 } \\ \text { Pltp } & \text { Emid1 } & \text { Il1rn } \\ \text { Cxcl9 } & \text { Ccl5 } & \text { Igsf21 } \\ \text { Svep1 } & \text { Wisp2 } & \text { Hapln3 } \\ \text { Col1a1 } & \text { Ntf3 } & \text { Angpt1 } \\ \text { Apod } & \text { Trem2 } & \text { Gdf3 } \\ \text { Figf } & \text { Itgbl1 } & \text { Bdnf } \\ \text { Erlec1 } & \text { Pcdh17 } & \text { Gzma } \\ \text { Lgmn } & \text { Cxcl14 } & \text { Xcl1 }\end{array}$

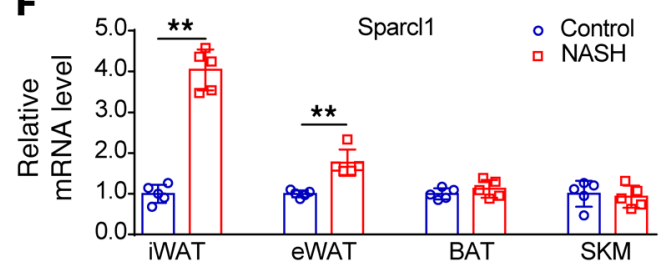

G
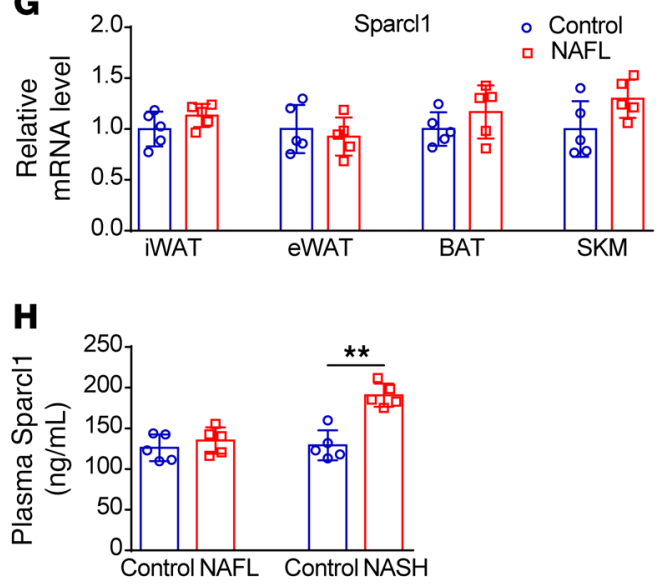

Figure 1. Sparcl1 is increased in the WAT of NASH mice. (A) Dysregulated genes (DEGs) in the eWAT of mouse models of NAFL and NASH. (B) Venn diagram of the DEGs from NAFL versus control and NASH versus control. (C) Functional enrichment analysis of DEGs in NASH mice. (D and E) Venn diagrams comparing the DEGs of NASH mice with a mouse secretome gene set (GEO GSE10246). (F and G) Relative mRNA levels of Sparcl1 from NASH (F) and NAFL (G) mice. iWAT, inguinal WAT; eWAT, epididymal WAT; BAT, brown adipose tissue; SKM, skeletal muscle. (H) ELISA analysis of plasma Sparcl1 concentrations in NAFL and NASH mice. $n=3$ per group $(\mathbf{A}-\mathbf{E})$ or 5 per group $(\mathbf{F}-\mathbf{H})$. Data are represented as mean \pm SEM. ${ }^{* *} P<0.01$ by 2 -tailed Student's $t$ test $(\mathbf{F}-\mathbf{H})$.

found that Sparcl1, a member of the Sparc family (23), was also increased (Figure 1D). Analysis by the cytoHubba plugin in Cytoscape also showed that Sparcl1 was involved in the top hub genes (Supplemental Figure 3C). Moreover, we found a correlation between Sparcl1 and the DEGs as illustrated by MCODE (Molecular Complex Detection) based on the PPI (protein-protein interaction) network (Supplemental Figure 3D). Collectively, these results suggest that Sparcll might be a candidate gene that exerts a pathogenic effect on the development of NASH.
Examination of the Sparcl1 expression pattern revealed that Sparcl1 mRNA was enriched in inguinal WAT (iWAT), eWAT, and brown adipose tissue (BAT), whereas its expression in other organs such as the liver and kidney was relatively low (Supplemental Figure 4A). Compared with stromal vascular fractions, Sparcl1 expression was enriched in mature adipocytes (Supplemental Figure 4B). Moreover, its mRNA level was highly induced during differentiation of 3T3-L1 white adipocytes along with PPAR $\gamma 2$, an established adipogenic marker (Supplemental Figure 4C). Con- 
sistent with the RNA-Seq data, our qRT-PCR analysis confirmed that the expression levels of Sparcl1 in iWAT and eWAT were significantly increased in NASH mice (Figure 1F), but remained unaltered in mice with NAFL (Figure 1G). Interestingly, upregulation of Sparcl1 was more evident in the iWAT of NASH mice than it was in the eWAT (Figure 1F). Although vAT has been confirmed to be associated with NASH development and progression $(14,15)$, our findings suggest that more attention should be paid to exploring the role of subcutaneous fat in chronic liver diseases. Furthermore, qRT-PCR revealed 1.42- and 2.17-fold upregulation of Sparcl1 mRNA levels in the livers of mice with NAFL or NASH, respectively (Supplemental Figure 4D), suggesting that the liver itself could produce Sparcl1 during development to NASH. In agreement, plasma Sparcl1 concentrations, measured by enzymelinked immunosorbent assays (ELISAs), were also selectively increased in NASH mice (Figure $1 \mathrm{H}$ ).

Plasma Sparcl1 levels were associated with hepatic pathological features in NASH patients. To determine the clinical relevance of our animal-based observations, plasma Sparcl1 levels were measured in normal individuals and liver biopsy-proven NAFLD patients (Supplemental Table 2). Plasma Sparcl1 levels were positively correlated with ALT, AST, and $\gamma$-glutamyl transferase (GGT) levels in the overall cohort (Figure 2, A-C), and gradually increased with the steatosis, ballooning, and lobular inflammation (Figure 2, D-F). We further distinguished NAFL and NASH patients according to the fatty liver inhibition of progression (FLIP) algorithm evaluated by a pathologist blinded to clinical data $(39,40)$. As a result, we also found that plasma Sparcl1 levels were significantly higher in NASH patients compared with normal individuals or NAFL patients (Figure 2G). To further evaluate the potential utility of Sparcl1 as a biomarker for the diagnosis of NASH, the area under the receiver-operating characteristic (AUROC) curve was calculated based on the FLIP algorithm. Plasma Sparcl1 exhibited a high accuracy for distinguishing patients with NASH from NAFLD patients (AUROC $=0.861, P<0.001$ ) and from the overall cohort (AUROC $=0.853, P<0.001$; Figure $2, \mathrm{H}$ and I). Moreover, the Sparcl1-ALT-AST model showed an improved accuracy in identifying NASH patients compared with the ALT-AST model (AUC $=0.859$ vs. 0.564, $P<0.001$; Figure 2J).

Acute injection of recombinant Sparcl1 protein activates the hepatic inflammatory response and promotes liver injury in steatotic mice. To study Sparcl1 function in NASH development, male C57BL/6J mice were fed an HFHC diet for 12 weeks to induce liver steatosis as mentioned above, and then intraperitoneally administered with a single treatment of recombinant Sparcl1 protein or vehicle control (saline). A dose of $0.2 \mathrm{mg} / \mathrm{kg}$ was chosen for these experiments because this dose can mimic the increased plasma levels of Sparcl1 observed in long-term HFHC diet-induced NASH mice (Supplemental Figure 5A). As a result, acute Sparcl1 treatment led to an increase in plasma ALT and AST levels in HFHC-fed mice (Supplemental Figure 5, B and C). Expression of hepatic inflammatory genes was dramatically increased in HFHC-fed mice treated with Sparcl1 (Supplemental Figure 5D). Plasma ALT and AST levels and expression of hepatic inflammatory genes were also strongly increased in HFD mice treated with Sparcl1 (Supplemental Figure $5, \mathrm{E}-\mathrm{G})$. However, treatment with Sparcl1 did not induce remarkable changes in the normal mice (Supplemental Figure 5, H-J). In addition, we found that Sparcl1 expression was not altered in other mouse models with liver injury: alcohol-treated mice (Supplemental Figure 6, A-F) and carbon tetrachloride-treated ( $\mathrm{CCl}_{4}$-treated) mice (Supplemental Figure 6, G-J). Acute Sparcl1 treatment had no additional effect on $\mathrm{CCl}_{4}$-induced liver injury and inflammatory response (Supplemental Figure 6, K-M). Collectively, these results suggest that Sparcl1 can activate the hepatic inflammatory response in the presence of steatosis, leading to liver injury and elevated expression of hepatic inflammatory cytokines.

Chronic exposure to Sparcl1 promotes hepatic steatosis and inflammation. The hepatic inflammatory response plays a central role in the initiation and progression of NASH (5). The finding that liver inflammation was activated in the acute Sparcl1-treated steatotic mice prompted us to explore the effects of chronic exposure to Sparcl1. Therefore, after 12 weeks of HFHC diet feeding, mice were intraperitoneally injected with saline or recombinant Sparcl1 protein $(0.2 \mathrm{mg} / \mathrm{kg})$ every other day for 3 weeks (Figure $3 \mathrm{~A})$. This treatment did not alter body weight, food intake, or fat content (data not shown). However, chronic exposure to Sparcl1 increased the liver/body weight ratio and hepatic TG content, accompanied with upregulation of lipogenic genes (Figure 3, B-D). Subsequent liver histology showed an increase in lipid accumulation in the livers of Sparcl1-treated mice compared with saline-treated mice (Figure 3E). Chronic Sparcl1 treatment resulted in the accumulation of F4/80-positive macrophages (Figure 3, F and $\mathrm{H}$ ), indicating enhanced hepatic inflammation. Moreover, hepatocyte death was observed by an increase in TUNEL staining in Sparcl1-treated mice (Figure 3, G and H), which was also reflected by elevated plasma ALT and AST levels (Figure 3I). Plasma concentrations of HMGB1, which is predominantly released from hepatocytes during liver injury (41), were also elevated in Sparcl1-treated mice (Figure 3J). Chronic Sparcl1 treatment also resulted in liver fibrosis, as shown by Sirius red staining and measurement of liver hydroxyproline content (Figure 3, K-M). In addition, mRNA expression of genes related to hepatic inflammation and liver fibrosis was upregulated in the livers of Sparcl1-treated mice (Figure 3N).

To further confirm the pathogenic effects of Sparcl1, we took advantage of adenoviral delivery techniques, which have been shown to efficiently upregulate genes of interest selectively in the WAT of mice (42-44). Given that the upregulation of Sparcl1 was more evident in the iWAT of NASH mice (Figure $1 \mathrm{~F}$ ), adenovirus expressing Sparcl1 (Ad-Sparcl1) or GFP was injected into iWAT of HFHC diet-fed steatotic mice. Forced expression of Sparcl1 in iWAT resulted in a 2.3-fold increase in plasma Sparcl1 levels (Supplemental Figure 7A). As a result, Sparcl1 overexpression increased hepatic TG content and plasma levels of liver injury makers in steatotic mice (Supplemental Figure 7, B-E). Abundance of F4/80-positive macrophages was increased accompanied with upregulation of genes involved in hepatic inflammation (Supplemental Figure 7, F and G). Consistently, hepatic hydroxyproline content, Sirius red staining, and mRNA expression of genes related to fibrosis were enhanced in Ad-Sparcl1-expressing mice (Supplemental Figure 7, H-J). Taken together, our findings clearly demonstrate that chronic exposure to Sparcl1 could accelerate the pathogenesis of NASH in the presence of steatosis.

Sparcl1 activates the hepatic inflammatory response through induction of CCL2. To elucidate the molecular basis of Sparcl1- 
A

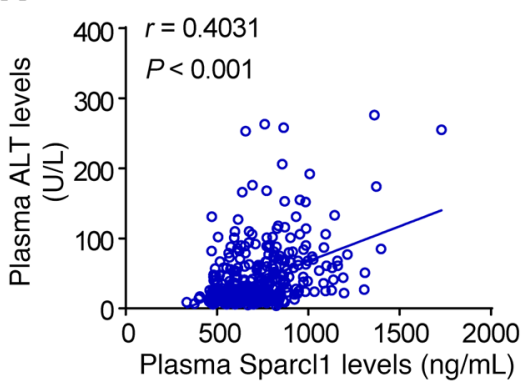

D

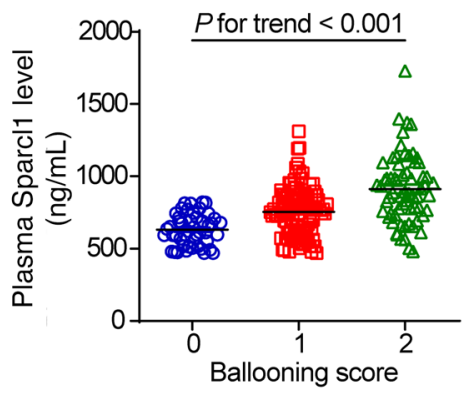

B

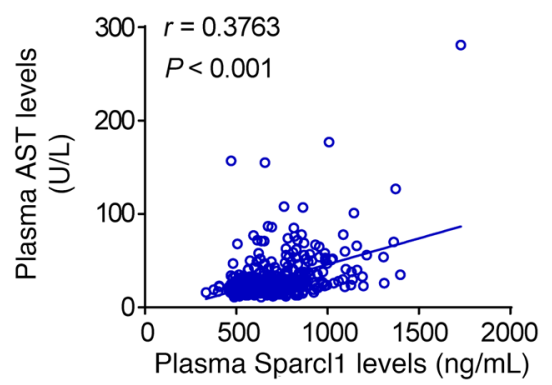

E

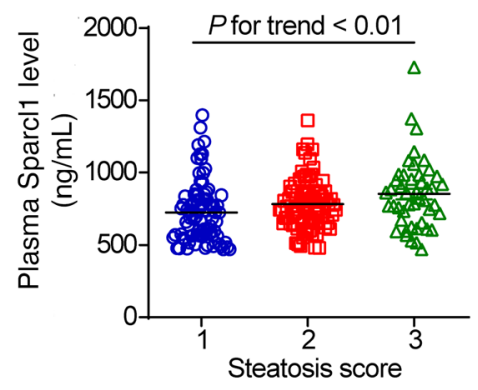

C

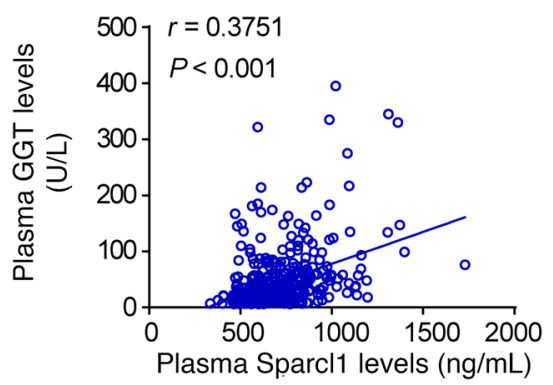

$\mathbf{F}$

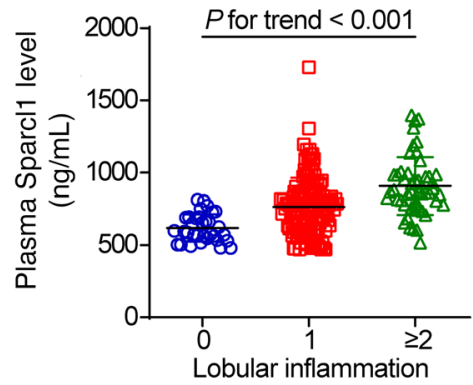

J

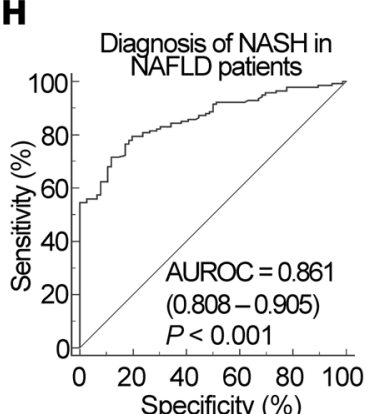

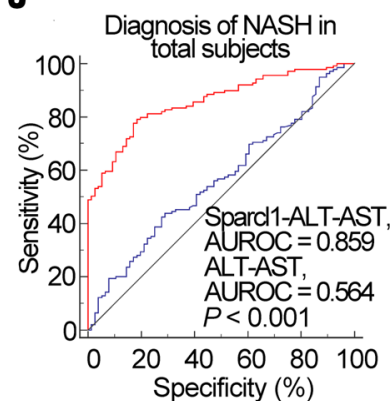

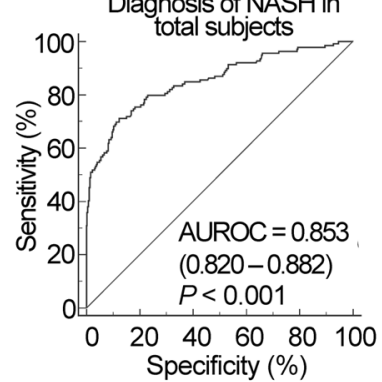

G

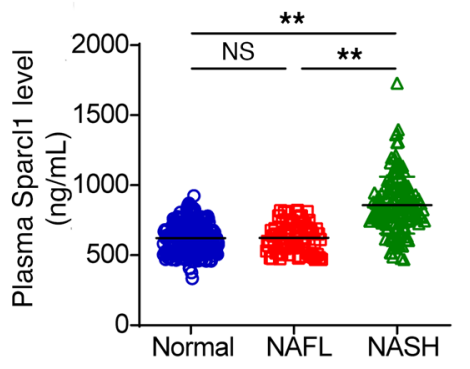

Figure 2. Association of plasma Sparcl1 levels with clinical features in patients. (A-C) Individual correlations between plasma Sparcl1 concentrations and ALT (A), AST (B), and GGT (C) levels. (D-F) Plasma Sparcl1 concentrations in patients with ballooning (D), steatosis (E), and lobular inflammation (F). (G) Plasma Sparcl1 concentrations in patients with NAFL $(n=76)$ or NASH $(n=141)$ and normal healthy individuals $(n=228)$. NAFLD patients were divided into NAFL and NASH according to the FLIP algorithm. ${ }^{* *} P<0.01$. (H-J) Sparcl1 diagnostic accuracy in humans. (H and I) NASH diagnostic accuracy in the NAFLD cohort (H) and the overall cohort (I). (J) Comparison of scores in prediction of NASH in the overall cohort. Red curve: Sparcl1-ALT-AST score. Blue curve: ALT-AST score. AUROC, area under the receiver-operating characteristic curve. Data are represented as mean \pm SEM. Significance was determined by Spearman's correlation (A-C), 1-way ANOVA (D-G), or DeLong's test (H-J).

mediated NASH progression, we conducted RNA-Seq analysis for DEGs using the livers of chronic recombinant Sparcl1 protein- or saline-treated mice (Supplemental Figure 8A). We found that 1888 genes were upregulated and 624 genes were downregulated (fold change $>1.5, P<0.05$; Supplemental Table 3). Among them, we identified a pronounced overexpression of CCL2, a member of the C-C chemokine family, in the livers of Sparcl1-treated mice (Supplemental Figure 8B). The increased expression of CCL2 in the livers and plasma was confirmed by qRT-PCR and ELISA analysis (Figure 4, A-C). In agreement, compared with normal or NAFL patients, plasma CCL2 concentrations were significantly increased in NASH patients (Figure 4D) and correlated with ALT, AST, and GGT levels (Figure 4, E-G). Importantly, plasma Sparcl1 and CCL2 levels were positively correlated in humans (Figure $4 \mathrm{H}$ ). We examined this further in Hepa1-6 cells, in which Sparcl1 alone failed to induce CCL2 expression but did so in the presence of palmitate (Sup- plemental Figure 8, C-E). However, preincubation with palmitate did not induce an inflammatory response or CCL2 expression in Hepa1-6 cells (Supplemental Figure 8, F-H). In addition, our RNA-Seq analysis found that $\mathrm{Cxcl1}, \mathrm{Cxcl} 2$, and $\mathrm{Cxcl} 5$ were also significantly upregulated in the livers of Sparcl1-treated mice (Supplemental Figure 8B and Supplemental Table 3), which was confirmed by qRT-PCR analysis (Figure 4I). These chemokines have been shown to mediate the recruitment of neutrophils through their common receptor CXCR2 $(45,46)$. Consistently, immunohistochemistry and qRT-PCR analysis showed that Ly6G, a neutrophil marker (47), was increased in the livers of mice with chronic Sparcl1 treatment (Figure 4, J and K). SB225002, a pharmacological inhibitor of CXCR2 (48), markedly inhibited Sparcl1-evoked neutrophil infiltration and Ly6G expression (Figure 4, L-N). Thus, our results indicate that hepatic infiltration of neutrophils might be, at least in part, attributable to the induction of Cxcl1, Cxcl2, and Cxcl5. 
A

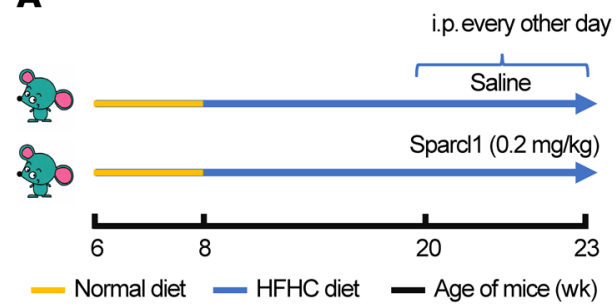

B

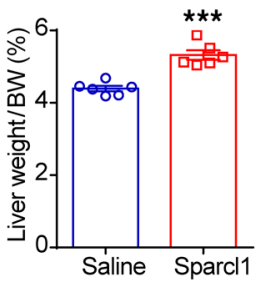

G TUNEL \& DAPI
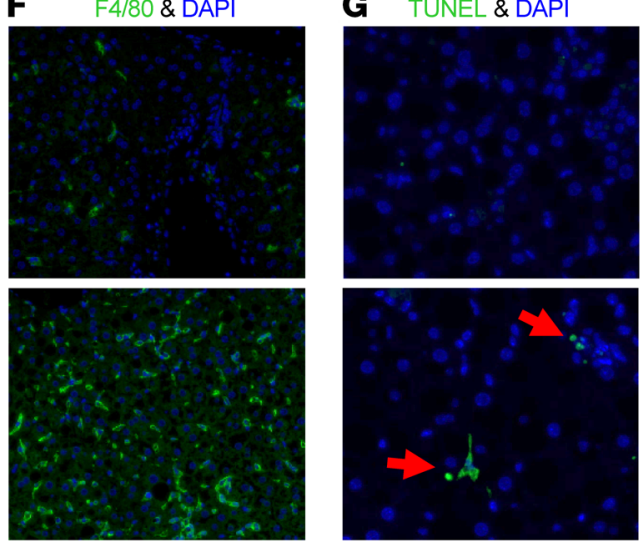

E
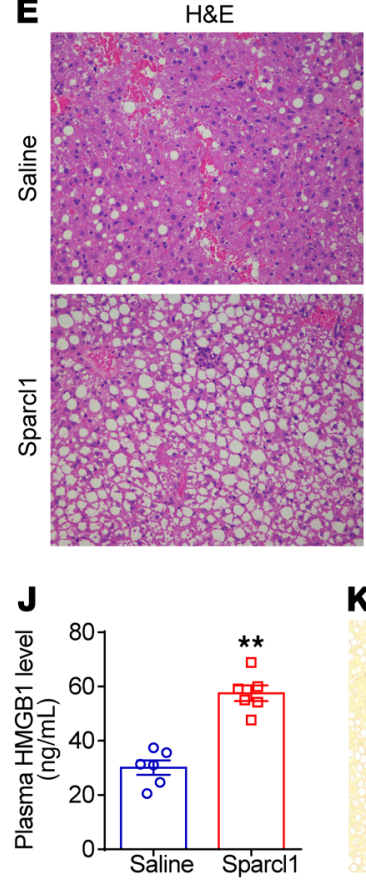

$\mathbf{K}$

Saline

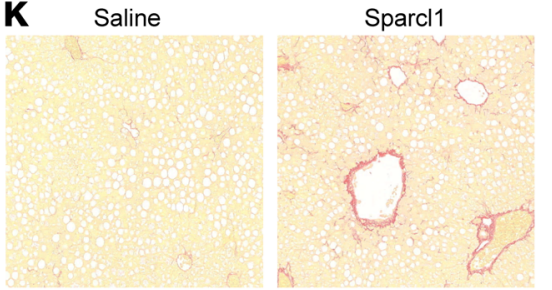

$\mathbf{N}$

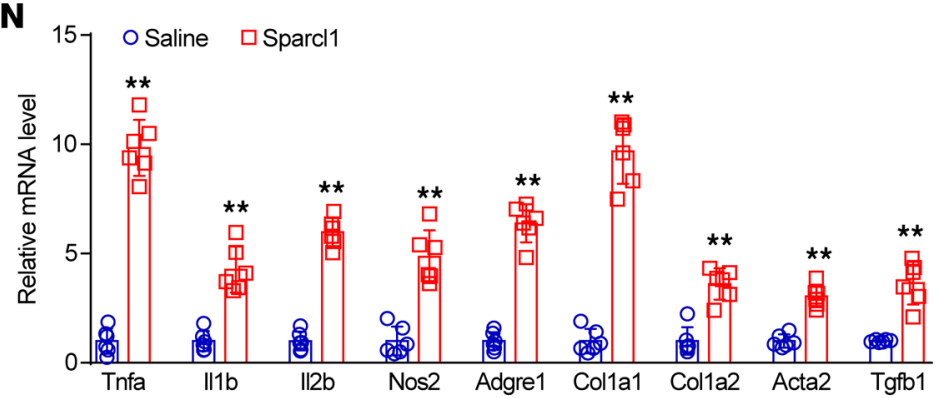

C

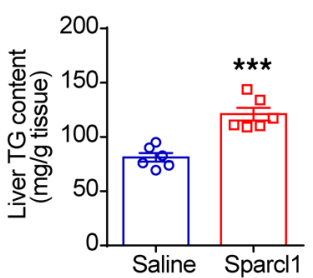

H
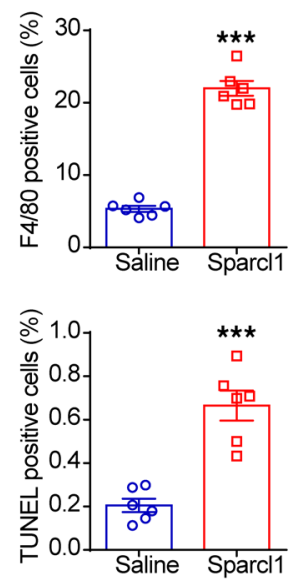

$\mathbf{L}$

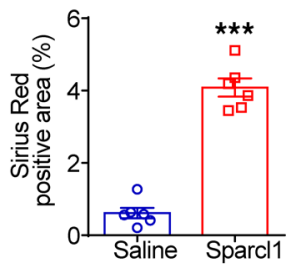

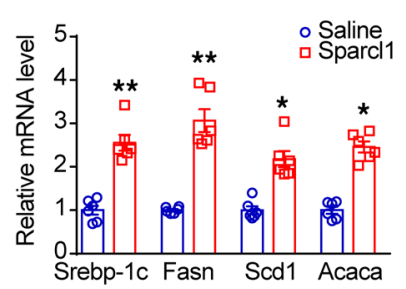

I
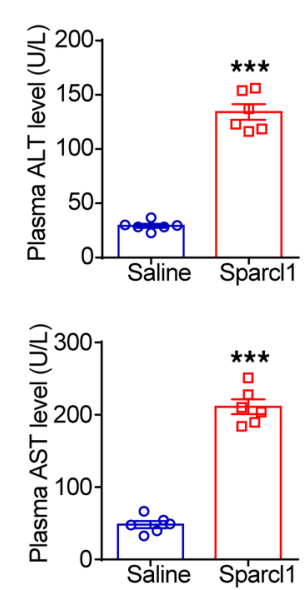

M

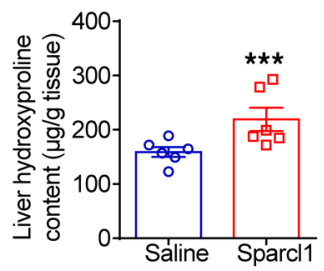

Figure 3. Chronic Sparcl1 treatment promotes NASH progression in mice. (A-N) C57BL/6) mice were fed an HFHC diet for 12 weeks, starting at 8 week of age. Then, mice were intraperitoneally injected with vehicle control (saline) or Sparcl1 $(0.2 \mathrm{mg} / \mathrm{kg}$ ) every other day for 3 weeks. $n=6$ per group. (A) Schematic of the experimental strategy. (B-D) Liver/body weight ratio (B), hepatic triglyceride content (C), and relative mRNA expression of lipogenic genes in livers of mice (D). (E-C) H\&E (E), F4/80 immunofluorescence (F), and TUNEL staining (G) of liver sections. (H) Quantification of the F4/80 and TUNEL staining images. (I and J) Plasma ALT and AST (I) and HMGB1 (J) levels. (K and L) Sirius red staining of liver sections (K) and quantification of images (L). (M) Liver hydroxyproline content. (N) Relative mRNA levels of genes involved in hepatic inflammation and fibrosis. Data are represented as mean \pm SEM. ${ }^{*} P<0.05,{ }^{* *} P<0.01,{ }^{* *} P<0.001$ by 2 -tailed Student's $t$ test (B-D, $\mathbf{H}-\mathbf{J}$, and $\left.\mathbf{L}-\mathbf{N}\right)$. Original magnification, $\times 200(\mathbf{E}-\mathbf{C}$ and $\mathbf{K})$.

To test whether CCL2 mediates the role of Sparcl1 in the activation of the hepatic inflammatory response, we used 3 approaches to block CCL2 function and then examined the effects of Sparcl1. First, we generated an adenoviral shRNA targeting CCL2 to inhibit its expression in the liver. HFHC-fed steatotic mice were administered with control shRNA or CCL2 shRNA and then injected with a single treatment of recombinant Sparcl1 protein or saline (Supplemental Figure 9, A and
B). As a result, knockdown of CCL2 substantially blocked the hepatic inflammatory response and liver injury induced by Sparcl1 treatment (Supplemental Figure 9, C-E). In addition, HFHC-fed mice were administered cenicriviroc, a pharmacological inhibitor of the CCL2 receptor $(49,50)$. Consistently, cenicriviroc also largely attenuated the ability of Sparcl1 to induce liver inflammation and injury in steatotic mice (Supplemental Figure 10, A-D). In addition, adeno-associated virus 8-mediated 
A
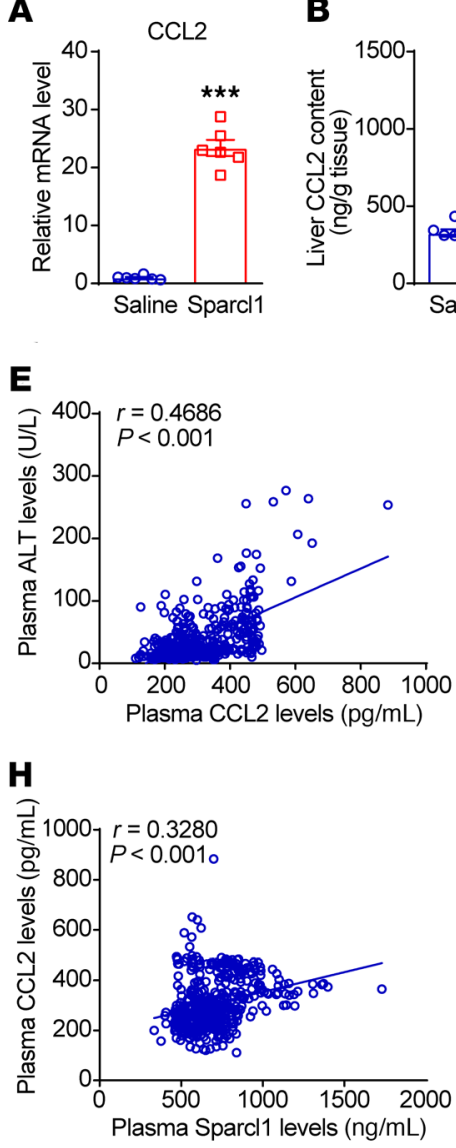

C

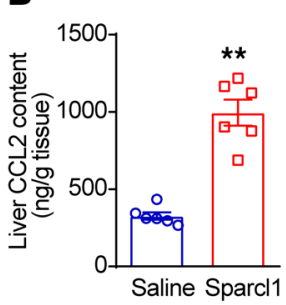

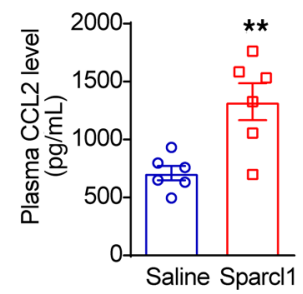

$\mathbf{F}$

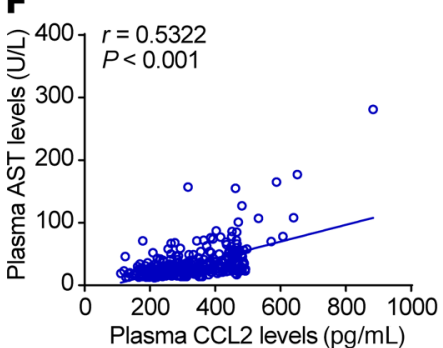

D

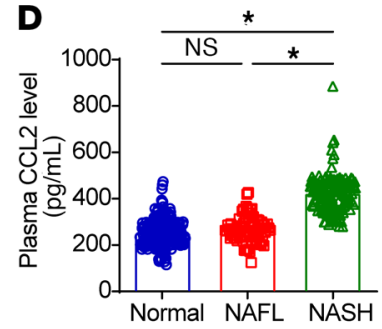

\section{I}

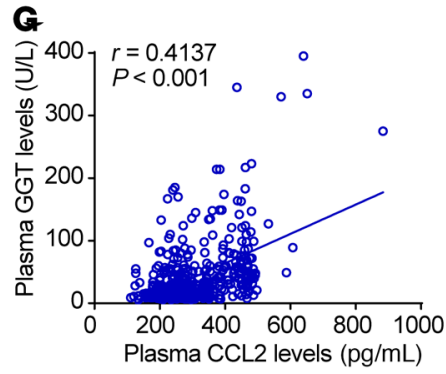

- Saline

․ Sparcl1

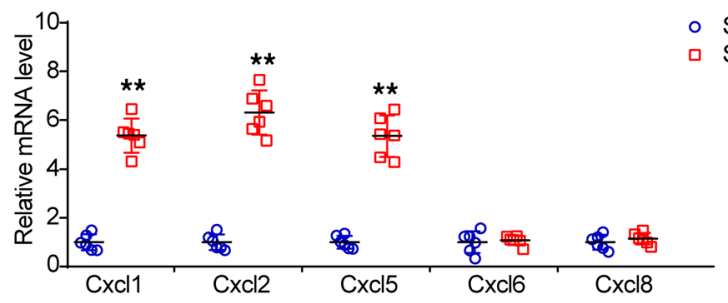

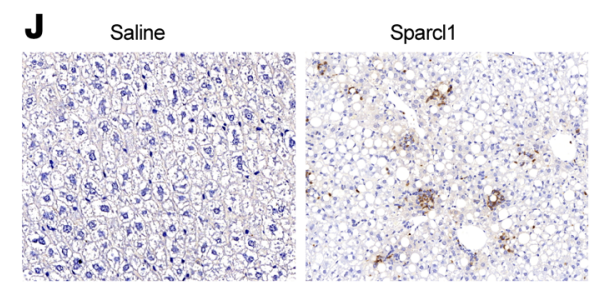

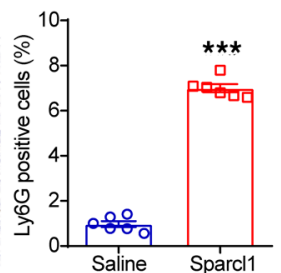

Sparcl1

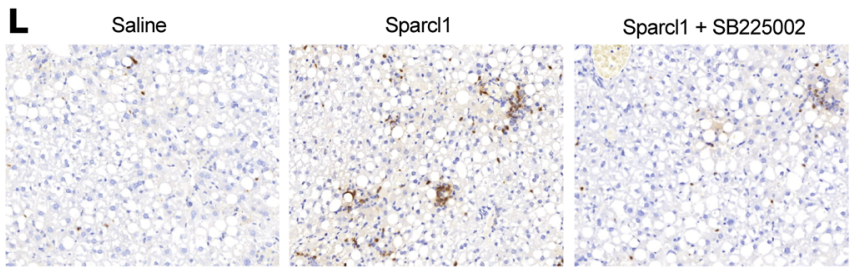

$\mathbf{K}$

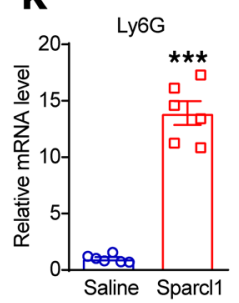

M

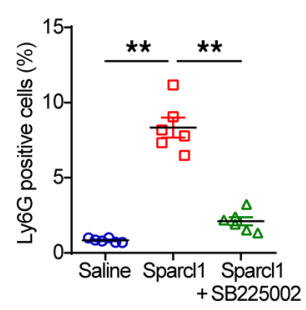

$\mathbf{N}$

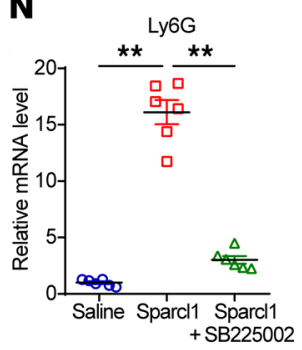

Figure 4. Sparcl1 induces CCL2 expression to promote NASH progression. (A and B) Relative mRNA levels (A) and protein concentrations (B) of CCL2 in the livers from mice chronically treated with saline or recombinant Sparcl1 protein. (C) Plasma CCL2 levels in the 2 groups of mice. (D) Plasma CCL2 concentrations in patients with NAFL $(n=76)$ or NASH $(n=141)$ and normal healthy individuals $(n=228)$. NAFLD patients were divided into NAFL and NASH according to the FLIP algorithm. (E-H) Individual correlations between circulating CCL2 concentrations and ALT (E), AST (F), GGT (C), and Sparcl1 (H) levels. (I) Relative mRNA levels of Cxcl1, Cxcl2, Cxcl5, Cxcl6, and Cxcl8 in the livers of the 2 groups of mice. (J) Ly6C staining of liver sections and quantification of images. (K) Relative mRNA levels of Ly6G in the livers from the 2 groups of mice. (L-N) C57BL/6) mice were fed an HFHC diet for 12 weeks, starting at 8 week of age. Then, mice were intraperitoneally injected with vehicle control (saline), Sparcl1 ( $0.2 \mathrm{mg} / \mathrm{kg}$ ), or Sparcl1 plus SB225002 (0.5 mg/kg) for 3 weeks. $n=6$ per group. Ly6G staining of liver sections $(\mathbf{L})$ and quantification of images $(\mathbf{M})$. Relative mRNA levels of Ly6G in the livers from 3 groups of mice $(\mathbf{N})$. $n=6$ per group (A-C and I-N). Data are represented as mean \pm SEM. ${ }^{*} P<0.05,{ }^{*} P<0.01,{ }^{* *} P<0.001$ by 2-tailed Student's $t$ test (A-C and $\left.\mathbf{I}-\mathbf{K}\right), 1$-way ANOVA (D, M, and $\mathbf{N})$, or Spearman's correlation $(\mathbf{E}-\mathbf{H})$. Original magnification, $\times 200$ (J and $\mathbf{L})$. 
A
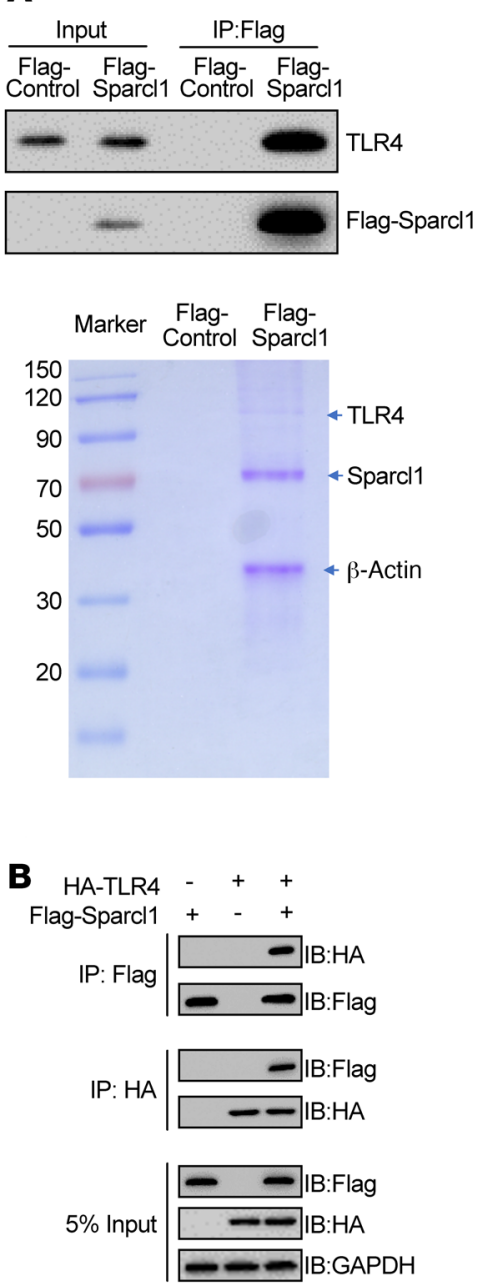

C

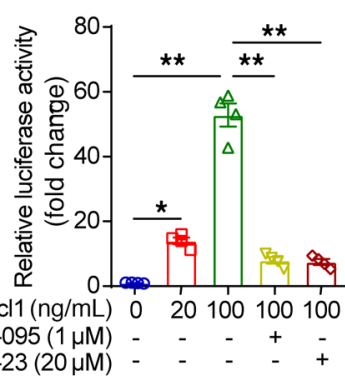

D

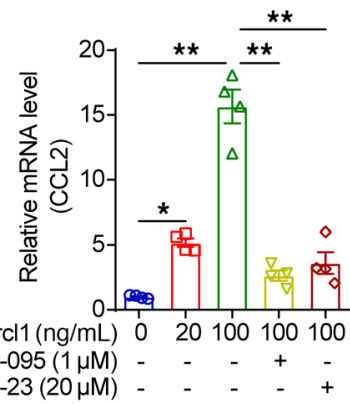

E

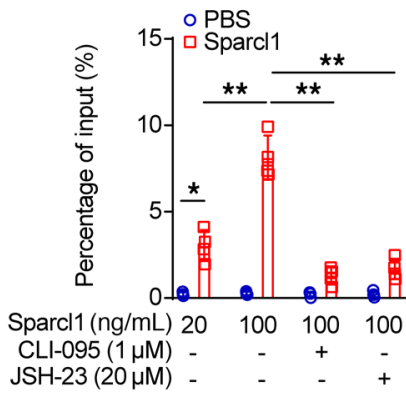

$\mathbf{F}$

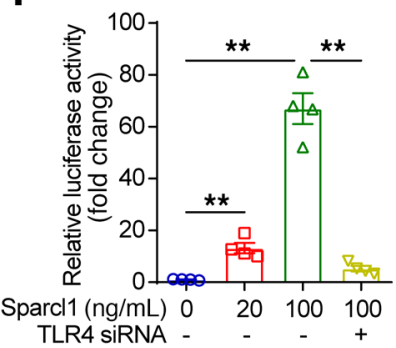

G

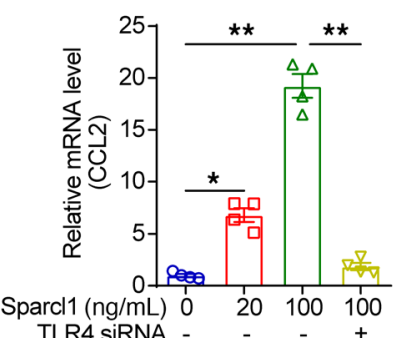

H

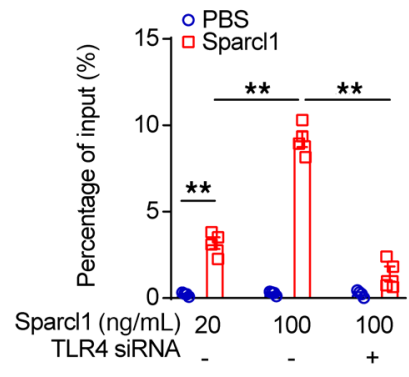

I

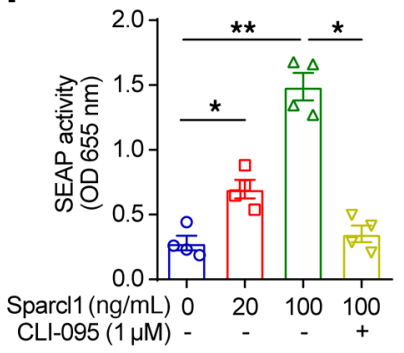

J

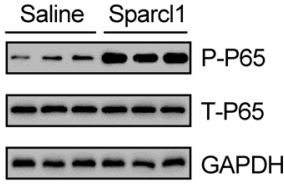

$\mathbf{K}$

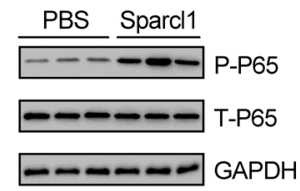

$\mathbf{L}$

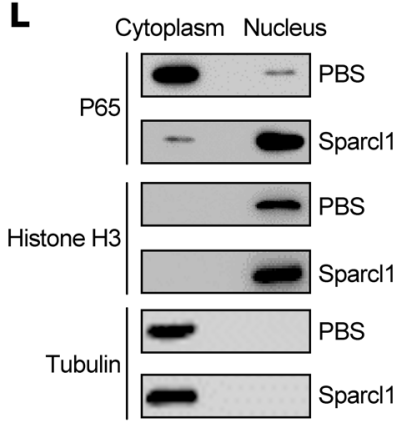

Figure 5. Sparcl1 activates TLR4 to upregulate CCL2 expression. Hepa1-6 cells were transfected with adenovirus expressing Flag-tagged vector control or Flag-tagged Sparcl1 for 36 hours. Protein lysates were immunoprecipitated using anti-Flag M2-agarose. The immunoprecipitates were then eluted with Flag peptide and immunoblotted using anti-Flag and anti-TLR4 antibodies. The immunoprecipitation of Flag-Sparcl1 interacting protein was visualized by Coomassie blue staining. (B) Protein-protein interaction of Sparcl1 and TLR4. HEK293T cells were transfected with Flag-tagged Sparcl1 and HA-tagged TLR4 for 48 hours. Cell lysates were immunoprecipitated with either anti-Flag antibody or anti-HA antibody, and the immunocomplexes were probed with the indicated antibodies. (C-E) Relative luciferase activity (C), relative mRNA level of CCL2 (D), and NF- $\mathrm{KB}$ binding to the CCL2 promoter (E) in Hepa1-6 cells after incubation of Sparcl1, in the absence or presence of CLI-095 $(1 \mu \mathrm{M})$ or JSH-23 (20 $\mu \mathrm{M})$. (F-H) Relative luciferase activity (F), relative mRNA level of CCL2 (G), and NF- $\mathrm{KB}$ binding to the CCL2 promoter (H) in Hepa1-6 cells after incubation with Sparcl1, in the absence or presence of TLR4 siRNA. (I) TLR4 activation in terms of SEAP activity in TLR4-MD2-overexpressing HEK Blue hTLR4 cells in response to Sparcl1 in the presence or absence of CLI-095. (J) Western blots showing phosphorylated p65 (P-P65) and total (T-P65) in the livers of HFHC diet-fed mice treated with Sparcl1 or saline. (K) Western blot showing phosphorylated p65 and total p65 in Hepa1-6 cells treated with Sparcl1 or saline. (L) Subcellular distribution of endogenous p65 in Hepa1-6 cells treated with Sparcl1 or vehicle control for 2 hours. (C-I and $\mathbf{K}-\mathbf{L})$ Hepa1-6 cells were preincubated with palmitate ( $0.2 \mathrm{mM})$ for 12 hours. Data are represented as mean \pm SEM. ${ }^{*} P<0.05,{ }^{*} P<0.01$ by 1 -way ANOVA $(\mathbf{C}-\mathbf{I})$.

(AAV8-mediated) knockdown of CCL2 was performed. After 12 weeks of HFHC diet feeding, mice were administered CCL2 shRNA or control shRNA through tail vein injection. One week later, mice were intraperitoneally injected with saline or recombinant Sparcl1 protein $(0.2 \mathrm{mg} / \mathrm{kg})$ every other day for another 3 weeks (Supplemental Figure 11, A-C). As a result, the pathogenic roles of Sparcl1 in NASH progression, including hepatic steatosis and inflammation, increased plasma levels of ALT, AST, and HMGB1, and changes in expression levels of genes involved in liver inflammation and fibrosis, were largely attenuated by knockdown of CCL2 (Supplemental Figure 11, D-J). Taken together, these findings suggest that Sparcl1 contributes to the hepatic inflammatory response and NASH progression, at least in part, through induction of CCL2.

Sparcl1 regulates CCL2 expression through binding to TLR4 and activation of the NF- $\kappa$ B pathway. Next, we investigated the molecular basis by which Sparcl1 upregulates CCL2 expression in hepatocytes. Hepa1-6 cells were transfected with adenovirus expressing Flag-tagged Sparcl1 or empty vector (control). Protein lysates were immunoprecipitated with anti-Flag M2-agarose. The immunoprecipitates were then eluted with Flag peptide, resolved by SDSPAGE, detected by Coomassie blue staining, and identified by liquid chromatography-tandem mass spectrometry (LC-MS/MS). Based on this analysis, TLR4, a pattern recognition receptor (10, 
A

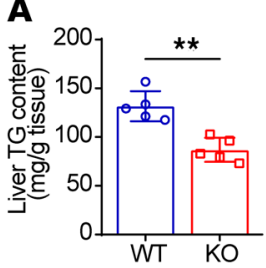

B

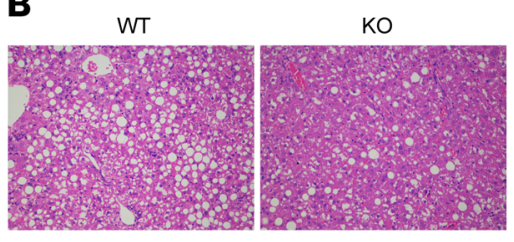

$\mathbf{F}$

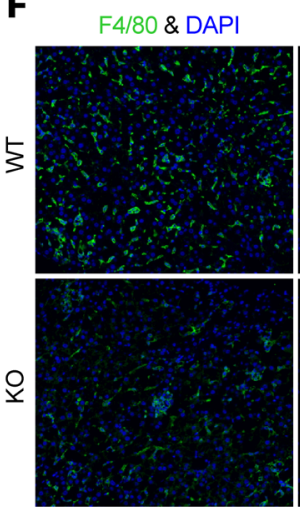

TUNEL \& DAPI

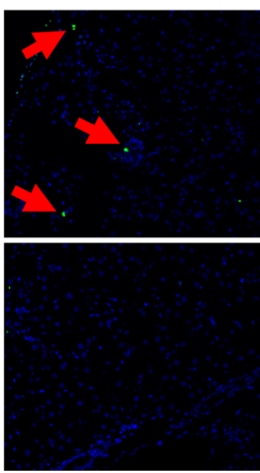

Ly6G

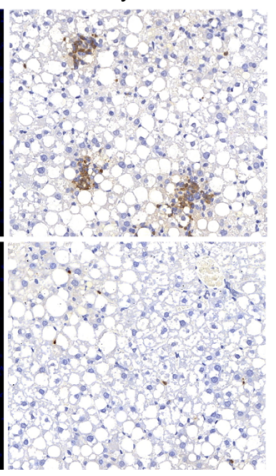

C

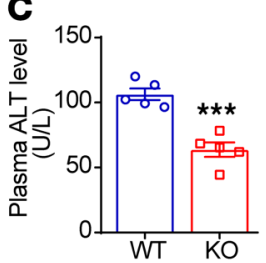

H

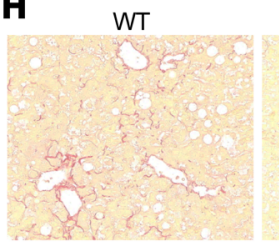

I

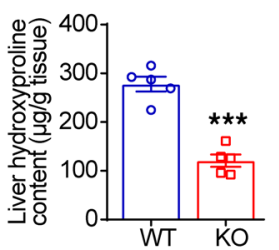

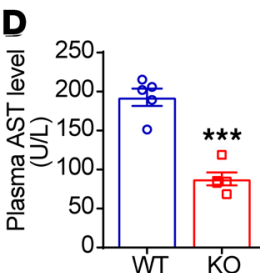

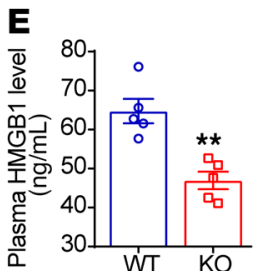

KO

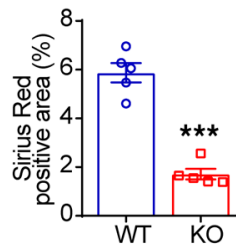

J

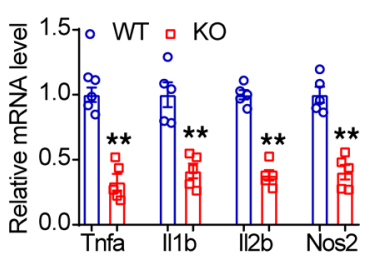

L

M
G

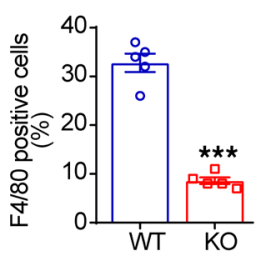

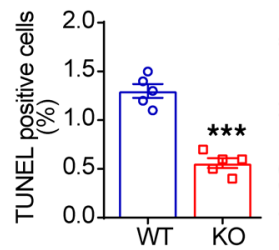

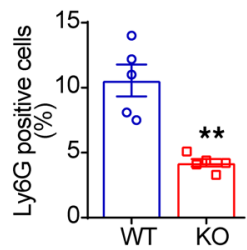

$\mathbf{K}$
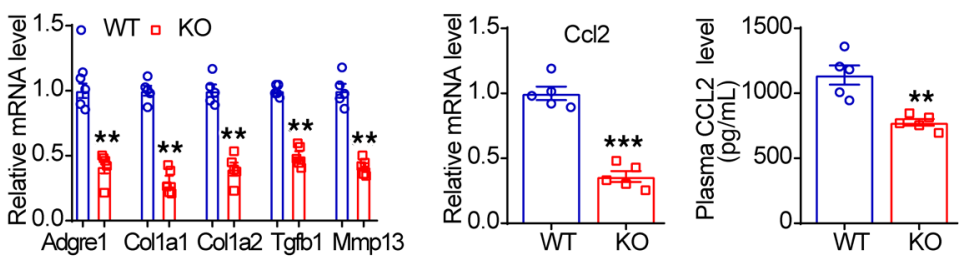

Figure 6. Sparcl1 deficiency protects mice from diet-induced NASH. Sparc/1 wild-type (WT) and knockout (KO) male mice were fed an HFHC diet for 28 weeks, starting at 8 weeks of age. $n=5$ per group. (A) Liver triglyceride (TC) content. (B) H\&E staining of liver sections. (C-E) Plasma levels of ALT (C), AST (D), and HMGB1 (E). (F) F4/80, TUNEL, and Ly6C staining of liver sections. (C) Quantification of the F4/80, TUNEL, and Ly6C staining images. (H) Sirius red staining and its quantification. (I) Liver hydroxyproline content. (J and $\mathbf{K}$ ) Relative mRNA levels of genes involved in liver inflammation and fibrosis. (L) Relative mRNA levels of CCL2 in the livers from the 2 groups of mice. (M) Plasma concentrations of CCL2 from the 2 groups of mice. Data are represented as mean \pm SEM. ${ }^{* *} P<0.01,{ }^{* *} P<0.001$ by 2 -tailed Student's $t$ test $(\mathbf{A}, \mathbf{C}-\mathbf{E}$, and $\mathbf{G}-\mathbf{M})$. Original magnification, $\times 200(\mathbf{B}, \mathbf{F}$, and $\mathbf{H})$.

11), was found to co-purify with Sparcl1 (Figure 5A). We confirmed the physical interaction of Sparcl1 with TLR4 in transiently transfected HEK293T cells using a coimmunoprecipitation approach (Figure 5B). We then tested the biological relevance of the Sparcl1TLR4 interaction. It has been well established that NF- $\mathrm{kB} / \mathrm{p} 65$ mediates TLR4 activation to regulate the transcription of multiple cytokines and chemokines, including CCL2 (51). Therefore, Hepa1-6 cells were incubated with Sparcl1 in the absence or presence of CLI-095 (a TLR4 signaling inhibitor) or JSH-23 (an NF-kB signaling inhibitor), followed by the examination of luciferase reporter assays, gene expression analysis, and chromatin immunoprecipitation (ChIP) assays. As a result, Sparcl1 could dosedependently activate the NF- $\mathrm{kB}$ luciferase reporter, upregulate the expression of CCL2, and enhance the occupancy of p65 on the CCL2 promoter (Figure 5, C-E). However, incubation with CLI-095 or JSH-23 largely attenuated the activation of NF- $\mathrm{KB}$ signaling by Sparcl1 treatment (Figure 5, C-E). In agreement, transfection of specific siRNA targeting TLR4 could also block Sparcl1's activation of the NF-KB reporter and induce CCL2 expression (Figure 5, F-H). Additionally, we employed genetically engineered
HEK Blue hTLR4 cells, which contain a TLR4/NF- $\kappa B-s e c r e t e d$ embryonic alkaline phosphatase (SEAP) reporter system to detect TLR4/NF-KB activation. As expected, incubation with Sparcl1 enhanced SEAP activity, which was inhibited by CLI-095 (Figure 5I). Finally, phosphorylation of p65 was increased in the livers of mice or Hepa1-6 cells treated with Sparcl1 (Figure 5J and 5K). Consistently, in response to Sparcl1 treatment, endogenous p65 was translocated into the nucleus in Hepa1-6 cells (Figure 5L). Collectively, our results suggest that Sparcl1 could upregulate CCL2 expression through activation of TLR4/NF- $\mathrm{kB}$ signaling.

Sparcl1 silencing suppresses hepatic steatosis, inflammation, and fibrosis. Having established that Sparcl1 upregulation is causally linked to the development of NASH, we next explored whether inhibition of Sparcl1 is sufficient to ameliorate diet-induced NASH in mice. To test this hypothesis, Sparcl1-knockout (Sparcl1-KO) mice were generated and their wild-type (WT) littermates were utilized as controls (Supplemental Figure 12, A-D). Under normal chow diet, there was no significant difference in the body weight, food intake, body composition, and hepatic TG content between WT and Sparcl1-KO mice (Supplemental Figure 12, E-H). We then 
A
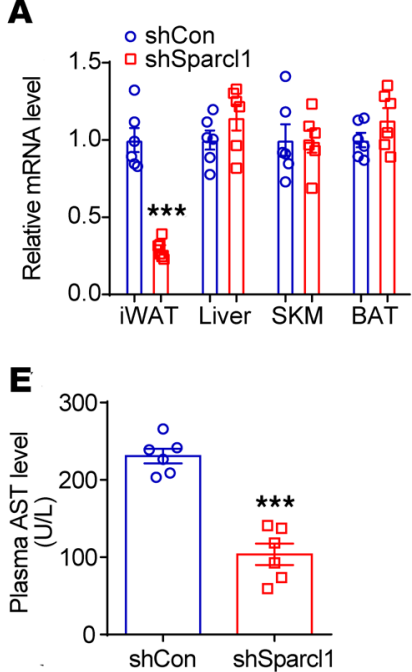

B
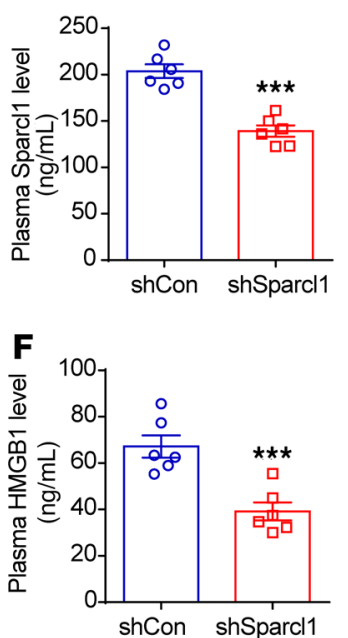

H
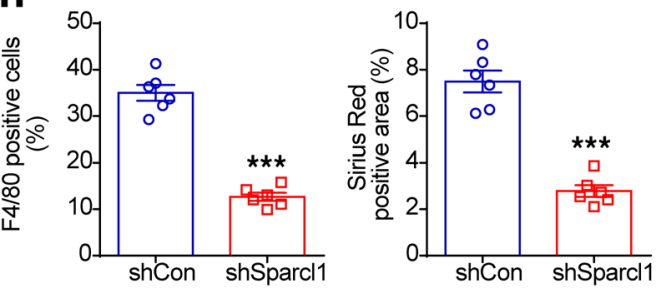

I

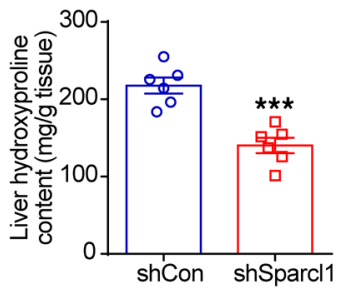

$\mathbf{L}$

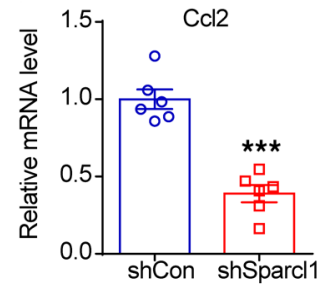

M

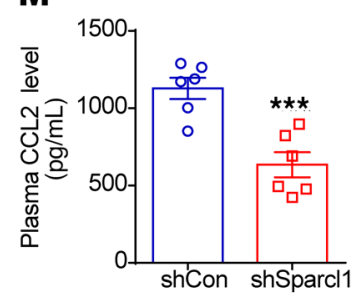

C
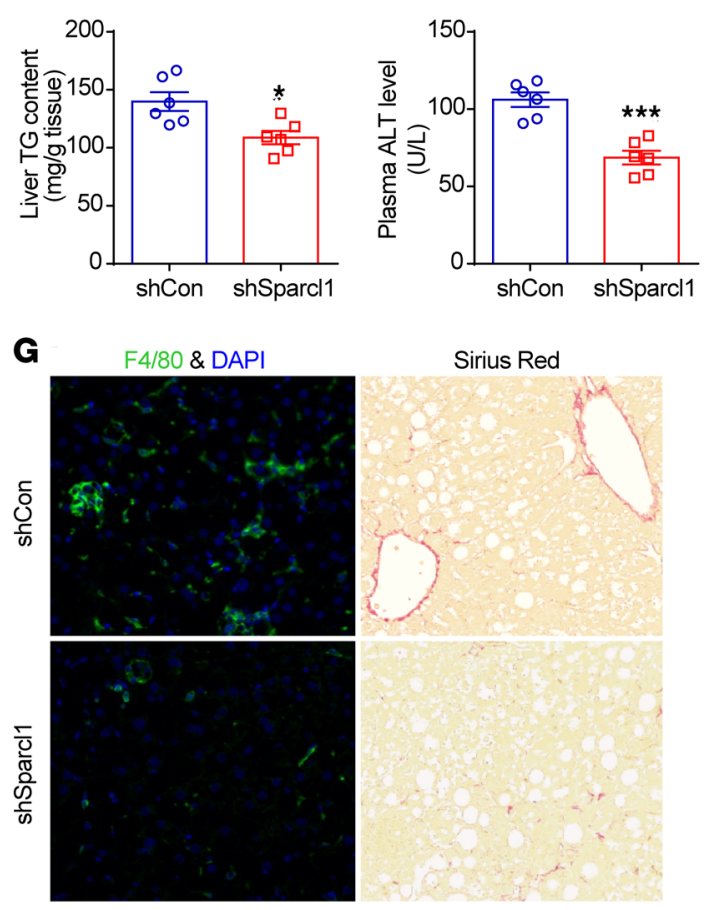

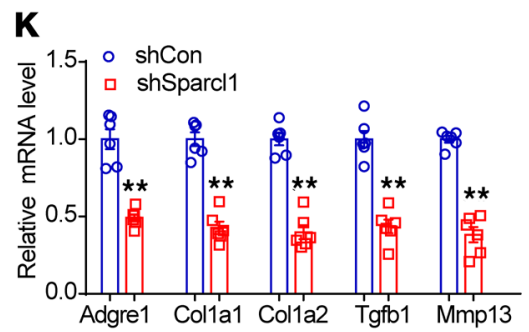

Figure 7. Knockdown of Sparcl1 in iWAT improves NASH pathogenesis in mice. (A-M) [57BL/6) mice were fed an HFHC diet for 28 weeks, starting at 8 week of age. Then, $5 \times 10^{10} \mathrm{pfu}$ of either shCon (AAV-scrambled shRNA) or shSparcl1 (AAV-Sparcl1 shRNA) was injected bilaterally into the inguinal fat pads of mice for 4 weeks. $n=6$ per group. (A) Relative mRNA levels of Sparcl1 in the iWAT, liver, SKM, and BAT from the 2 groups of mice. (B) Plasma Sparcl1 concentrations. (C) Liver triglyceride (TC) content. (D-F) Plasma levels of ALT (D), AST (E), and HMGB1 (F). (C) F4/80 and Sirius red staining of liver sections. (H) Quantification of the F4/80 and Sirius red staining images. (I) Liver hydroxyproline content. (J and K) Relative mRNA levels of genes involved in liver inflammation and fibrosis. (L) Relative mRNA levels of CCL2 in the livers from the 2 groups of mice. (M) Plasma concentrations of CCL2 from the 2 groups of mice. Data are represented as mean \pm SEM. ${ }^{*} P<0.05,{ }^{* *} P<0.01,{ }^{* *} P<0.001$ by 2 -tailed Student's $t$ test $(\mathbf{A}-\mathbf{F}$ and $\mathbf{H}-\mathbf{M})$. Original magnification, $\times 200$ (G).

fed WT and Sparcl1-KO mice with HFHC diet for 12 weeks or 28 weeks. Under 12 weeks of feeding, hepatic TG content, plasma ALT and AST levels, and mRNA expression of genes involved in liver inflammation and fibrosis were similar between the 2 groups of mice (Supplemental Figure 13, A-E). However, after feeding HFHC diet for 28 weeks, Sparcl1-deficient mice had lower liver
TG content, reduced lipid droplets in hepatocytes, and lower plasma ALT, AST, and HMGB1 levels (Figure 6, A-E). Immunofluorescence staining revealed that the abundance of $\mathrm{F} 4 / 80$ macrophages, TUNEL-positive hepatocytes, and infiltration of neutrophils were lower in the Sparcl1-KO mice (Figure 6, F and $\mathrm{G})$, indicative of attenuated liver inflammation. In support of this, 
A

Recombinant Sparcl1 protein $(\mu \mathrm{g})$

\begin{tabular}{|lll}
0.5 & 1.0 & 2.0 \\
\hline & & \\
& & \\
\end{tabular}

B

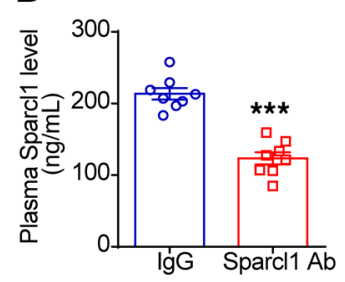

C

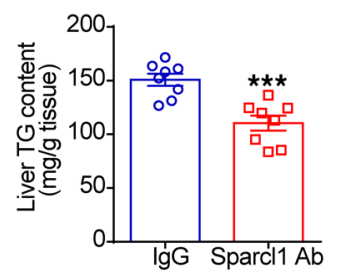

D

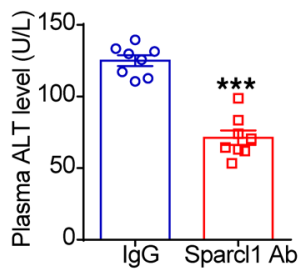

$\mathbf{E}$

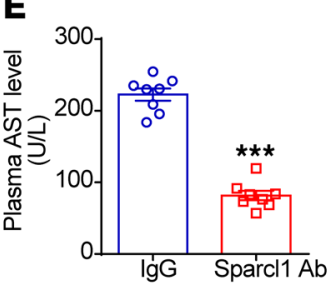

H

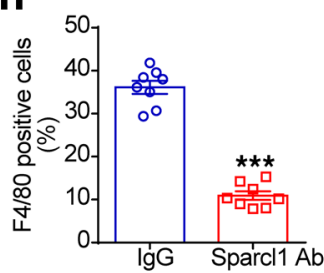

I

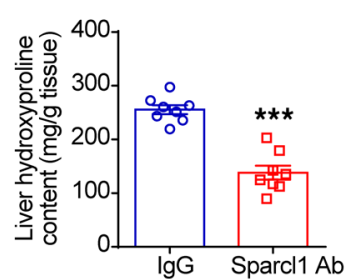

$\mathbf{L}$

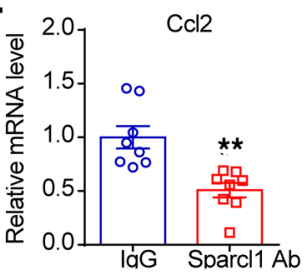

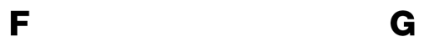

G $\quad F 4 / 80 \& D A P I$
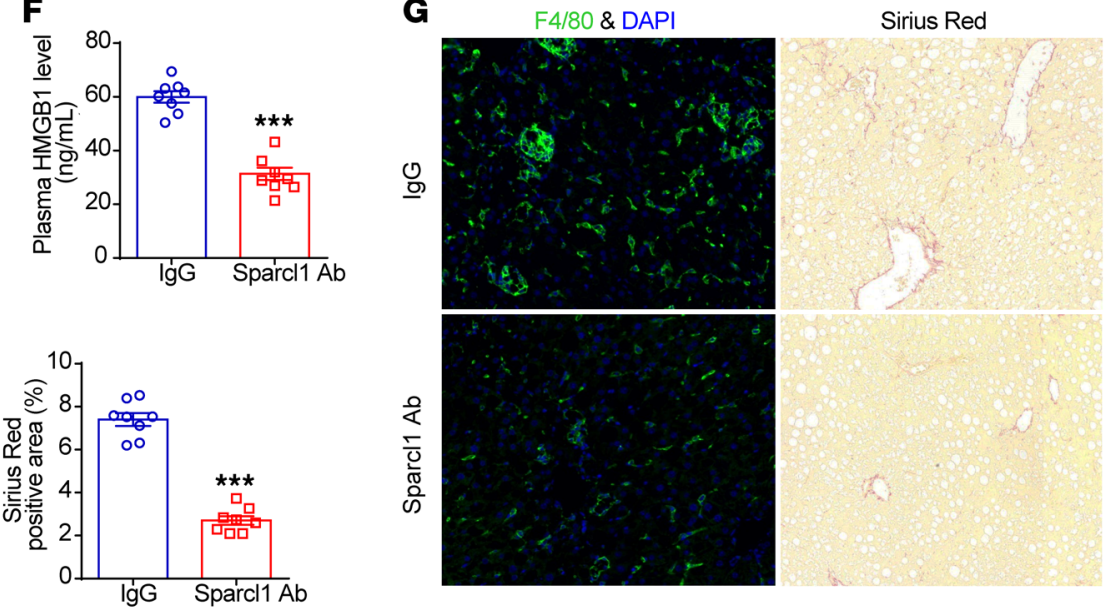

K
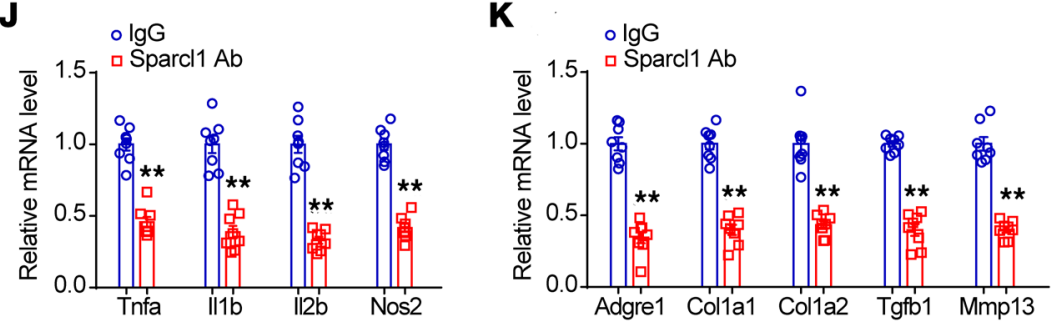

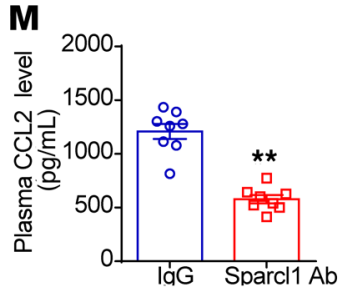

Figure 8. Neutralization of Sparcl1 improves NASH pathogenesis in mice. (A-M) C57BL/6) mice were fed an HFHC-diet for 28 weeks, starting at 8 week of age. Then, mice were injected intravenously with IgG or anti-Sparcl1 antibody (5 mg/kg) twice per week for 4 weeks. $n=8$ per group. (A) Confirmation of anti-Sparcl1 antibody binding by Western blotting. (B) Plasma Sparcl1 concentrations in the 2 groups of mice. (C) Liver triglyceride (TC) content. (D-F) Plasma levels of ALT (D), AST (E), and HMCB1 (F). (G) F4/80 and Sirius red staining of liver sections. (H) Quantification of the F4/80 and Sirius red staining images. (I) Liver hydroxyproline content. (J and K) Relative mRNA levels of genes involved in liver inflammation and fibrosis. (L) Relative mRNA levels of CCL2 in the livers from the 2 groups of mice. (M) Plasma concentrations of CCL2 from the 2 groups of mice. Data are represented as mean $\pm S E M$. ${ }^{* *} P<$ $0.01,{ }^{* *} P<0.001$ by 2 -tailed Student's $t$ test $(\mathbf{B}-\mathbf{F}$ and $\mathbf{H}-\mathbf{M})$. Original magnification, $\times 200$ (C).

liver fibrosis and hepatic hydroxyproline content were reduced in the Sparcl1-KO mice (Figure 6, H and I). Consistently, mRNA expression of genes involved in inflammation and fibrosis was markedly downregulated in the livers of knockouts (Figure 6, J and $\mathrm{K})$. In addition, hepatic mRNA expression and plasma concentrations of CCL2 were lower in the knockouts (Figure 6, L and M). To further confirm these results, 2 groups of mice were fed with HFHC diet for 36 weeks. As a result, Sparcl1 deficiency protected mice from prolonged-time HFHC diet feeding-induced key pathogenic events of NASH and liver fibrosis (Supplemental Figure 14, A-L).
Recent studies have shown that viral delivery techniques could efficiently downregulate genes of interest selectively in the iWAT of mice (52-55). Therefore, AAV8-shRNA targeting Sparcl1 or negative control (scrambled shRNA) were generated and injected into iWAT depots of HFHC diet-induced NASH mice. As a result, Sparcl1 shRNA treatment reduced Sparcl1 mRNA expression in the iWAT and plasma Sparcl1 concentrations, compared with negative control shRNA-injected mice (Figure 7, A and B). In agreement, metabolic studies and gene expression analysis showed that shRNA-mediated knockdown of Sparcl1 improved NASH patho- 
A
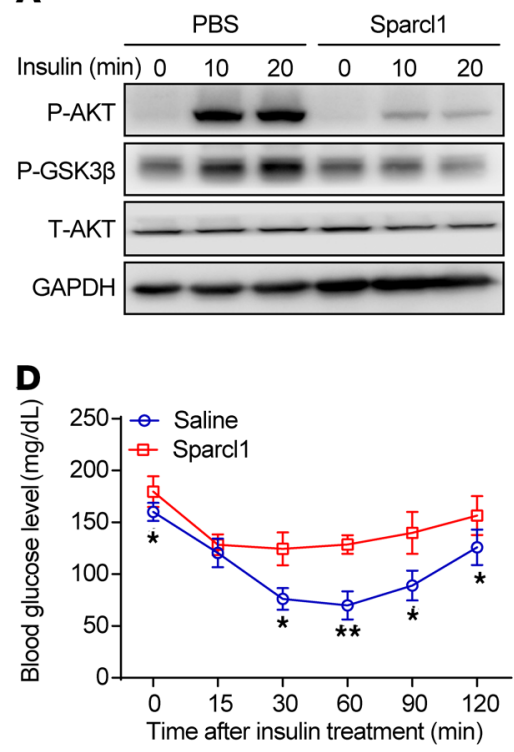

G

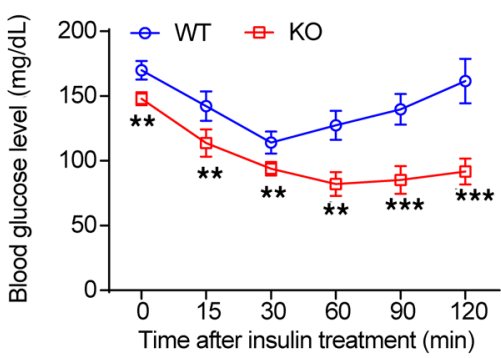

B

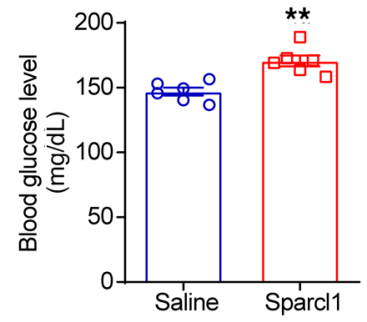

E

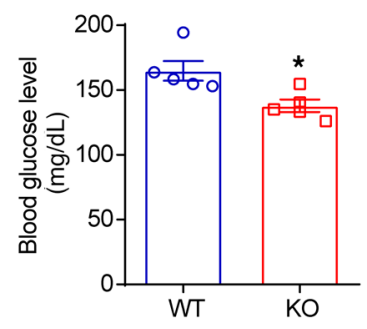

C

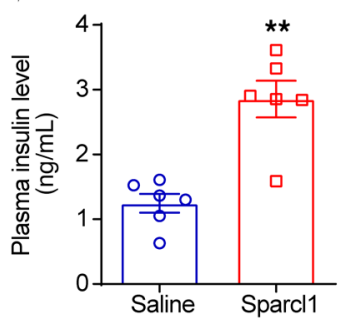

$\mathbf{F}$

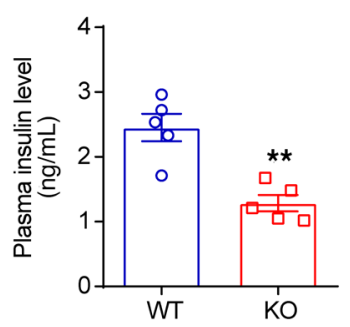

H

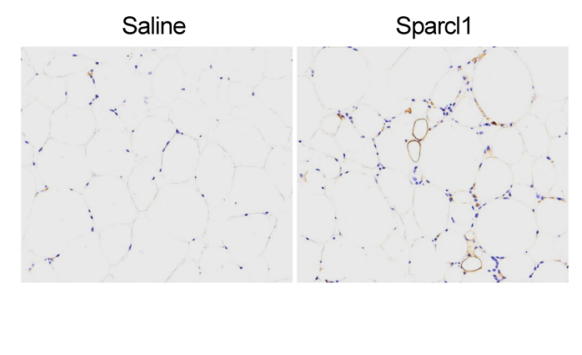

I

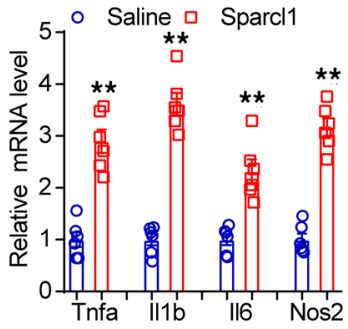

Figure 9. Sparcl1 disrupts systemic glucose metabolism in NASH mice. (A) Western blots showing the insulin-stimulated phosphorylated AKT (P-AKT) and GSK3 $\beta$ (P-CSK3 $\beta$ ) in Hepa1-6 cells treated with Sparcl1 $(100 \mathrm{ng} / \mathrm{mL}$ ) or vehicle control (PBS). Cells were preincubated with palmitate (0.2 mM) for 12 hours. (B-D) Blood glucose levels (B), plasma insulin levels (C), and insulin tolerance test (D) in HFHC diet-fed mice with chronic treatment of Sparcl1 or saline. $n=6$ per group. (E-C) Blood glucose levels (E), plasma insulin levels (F), and insulin tolerance test (C) in Sparcl1-WT and -KO mice fed an HFHC diet for 28 weeks. $n=5$ per group. (H) F4/80 crown-like structures in the iWAT sections from HFHC diet-fed mice after chronic treatment with Sparcl1 or saline. (I) Relative mRNA levels of proinflammatory cytokines. $n=6$ per group. Data are represented as mean $\pm \mathrm{SEM} .{ }^{*} P<0.05,{ }^{* *} P<0.01,{ }^{* * *} P<0.001$ by 2 -tailed Student's $t$ test (B, C, E, F, and I) or 1-way ANOVA (D and G). Original magnification, $\times 200$ (H).

genesis and reduced CCL2 expression in NASH mice (Figure 7, $\mathrm{C}-\mathrm{M})$. These findings further demonstrated that WAT might be an important source of Sparcl1 during development to NASH.

Finally, to evaluate in vivo the potential therapeutic effects of suppressing Sparcl1 in treating NASH, we developed a neutralizing antibody to interfere with Sparcl1 and administered it to HFHC diet-induced NASH mice. According to the methods described in recent studies (56-58), NASH mice were treated with either anti-Sparcl1 neutralizing antibody or control IgG antibody twice per week for 4 weeks. The specificity and efficiency of the neutralizing antibody were confirmed by Western blotting and ELISA, which showed that plasma Sparcl1 concentrations were markedly reduced by this treatment (Figure 8, A and B). Compared with NASH mice treated with IgG controls, hepatic TG content and plasma levels of ALT, AST, and HMGB1 were markedly decreased in NASH mice treated with Sparcl1-neutralizing antibody (Figure 8, $\mathrm{C}-\mathrm{F})$. In addition, the abundance of $\mathrm{F} 4 / 80$-positive macrophages, liver fibrosis, and hepatic hydroxyproline content were reduced (Figure 8, G-I). In agreement, expression of genes involved in hepatic inflammation and fibrosis was downregulated (Figure
8, J and K). Additionally, mRNA expression and plasma levels of CCL2 were reduced (Figure 8, L and M). Collectively, these results demonstrated that inhibition of Sparcl1 with AAV8-shRNA or a neutralizing antibody could improve NASH progression in mouse models, suggesting that targeting Sparcl1 might be an effective therapeutic strategy for NASH.

Sparcl affects systemic metabolism at the whole-body level. Activation of TLR4/NF- $\mathrm{BB}$ signaling in hepatocytes has been shown to affect systemic metabolic homeostasis, resulting in hyperglycemia and insulin resistance $(59,60)$. Consistent with this hypothesis, we found that insulin-stimulated phosphorylation of AKT and its target protein, GSK3 $\beta$, was repressed by Sparcl1 treatment in Hepa1-6 cells (Figure 9A). Chronic injection of recombinant Sparcl1 also induced fasting hyperglycemia and hyperinsulinemia and impaired insulin sensitivity in HFHC diet-fed mice (Figure 9, B-D), whereas Sparcl1-KO mice were partially protected from long-term HFHC diet-induced abnormal glucose homeostasis (Figure 9, E-G). Furthermore, Sparcl1 treatment could promote adipose tissue inflammation, as shown by crown-like structure formation and upregulation of inflammatory genes (Figure 9, $\mathrm{H}$ 
and I). Therefore, Sparcl1 may have systemic effects at the wholebody level. Moreover, our results support the notion that NASH can further disrupt systemic glucose metabolism in the context of obesity or overnutrition (61).

\section{Discussion}

Typically, NASH is characterized by the presence of sustained hepatic inflammation, which is strongly associated with liver injury and fibrosis. Previous studies showed that inflammation can induce lipid accumulation during NASH progression and may precede steatosis $(2,5,62)$, while alleviation of liver inflammation could improve hepatic TG retention and steatosis $(63,64)$. For instance, activation of hepatocyte TLR4 signaling due to Tmbim1 deficiency substantially increased hepatic inflammation in mice fed an HFD for 24 weeks (63). As a result, hepatic TG content was increased in the Tmbim1-deficient mice. On the other hand, suppression of hepatocyte TLR 4 signaling by Tmbim1 overexpression alleviated inflammation and reduced steatosis in mice fed a long-term HFD (63). Moreover, Guo et al. showed that adipose tissue-enriched endocrine factor $\mathrm{Nrg} 4$ could reduce hepatic inflammation and preserve hepatocyte health (22). As a result, Nrg4 deficiency exacerbated HFHC diet-induced hepatic TG retention, while overexpression of $\mathrm{Nrg} 4$ protected mice from long-term HFHC diet-induced steatosis and inflammation (22). Therefore, these results support the notion that hepatic steatosis and inflammation are tightly associated in NASH progression. Nevertheless, the critical factors that drive the hepatic inflammatory response in NASH remain poorly understood. Initially, a 2-hit hypothesis was posited decades ago, which described that the first hit is steatosis while the second hit is from oxidative stress (65). However, this view is now considered outdated $(2,3)$. A multiple-hit hypothesis involving a myriad of factors has been proposed, which includes lipotoxicity, insulin resistance, hepatocyte inflammasome activation, and alterations in gut microbiota $(2,3)$. Therefore, both the pathogenic drivers and molecular basis that underlie NASH progression are complicated and require more intensive studies. In this study, using HFHC diet-induced mouse models that encompass 2 time periods, we compared the WAT transcriptome and identified 118 cytokines selectively dysregulated in the WAT of NASH mice. Some of these cytokines have been shown to regulate adipogenesis (such as Wnt1Ob and Angptl2), adipose inflammation (such as Gdf3 and Cyr61), and fat mass and energy expenditure (such as Fgf1O and Vegfb) in adipose tissues (66-71). Whether they play a role in the development of NASH through direct or indirect mechanisms remains poorly understood. Therefore, our transcriptome screening may provide a valuable resource to explore the molecular mechanisms of the WAT/liver endocrine axis underlying NASH progression for future studies.

We further investigated the pathogenic roles of Sparcl1 in $\mathrm{NASH}$ progression through several approaches. Both chronic recombinant Sparcl1 protein injection and adenovirus-mediated overexpression of Sparcl1 led to a hepatic inflammatory response and liver injury in mice in the presence of simple steatosis, as evidenced by elevated plasma concentrations of ALT, AST, and HMGB1. In addition, liver fibrosis was induced by chronic Sparcl1 treatment, as shown by Sirius red staining and increased hepatic hydroxyproline content. Comparison of chronic Sparcl1-treated mice (Figure 3 and Supplemental Figure 7) to the 28-week HFHC diet-fed mice (Supplemental Figure 2) showed that the hepatic inflammatory response and liver injury were more severe in Sparcl1-treated mice. Therefore, exogenous Sparcl1 treatment in 12-week HFHC diet-fed mice can worsen liver disease toward NASH and achieve a similar state to that of the long-term HFHC diet-fed model. On the other hand, Sparcl1-deficient mice by gene knockout or knockdown were protected from long-term HFHC diet-induced NASH, as demonstrated by decreased hepatic steatosis and inflammation and reduced liver injury and fibrosis. Of pathophysiological significance, Sparcl1 expression was upregulated in the WAT of NASH mice, and plasma Sparcl1 concentrations were correlated with hepatic pathological features in NASH patients. Therefore, together with the use of neutralizing antibody, these findings suggest that blocking Sparcl1 signaling might be a promising therapeutic target for NASH treatment. Of note, our data showed that liver fibrosis could be reduced more than $50 \%$ after 4 weeks of treatment in mice, which was also observed in recent studies $\left(56^{-58}\right)$. One possible explanation is that the diet-induced NASH phenotypes, especially liver fibrosis, might be mild. The mild symptoms of liver fibrosis in NASH mice might be easily reversed or ameliorated. Indeed, the diet-based models are refractory to fully progressing to severe fibrosis, even after long-term feeding (72). Therefore, further studies are still needed to explore the therapeutic effects of Sparcl1-neutralizing antibody in NASH models with severe liver fibrosis. We also found that Sparcl1 mRNA levels were moderately increased in the livers of mice with simple steatosis or NASH (Supplemental Figure 3D). This is consistent with a recent study that showed a 2-fold upregulation of hepatic Sparcl1 mRNA expression in a genetic mouse model of steatohepatitis and fibrosis (73). Nevertheless, the basal expression level of Sparcl1 was very low in the murine liver (23), which was also observed in our study (Supplemental Figure 4A). In addition, Sparcl1 was found to be expressed in many organs such as the lung and gastrointestinal tract (23). Other tissue- and cell type-dependent expression of Sparcl1 may play a role in the $\mathrm{NASH}$ progression as well.

It has been well established that TLR4 is a critical link mediating obesity-associated hepatic steatosis and liver inflammation and injury (74). In the liver, TLR4 is functionally expressed in hepatocytes and macrophages/Kupffer cells $(63,75,76)$. Our experiments also showed that Sparcl1 could activate TLR4 signaling in RAW264.7 macrophage cells (Supplemental Figure 15, A and B). Therefore, we speculate that in addition to hepatocytes, Sparcl1 may also induce the production of inflammatory mediators through activation of TLR4 signaling in macrophages/Kupffer cells. However, through Kupffer cell depletion and bone marrow transplantation, Li et al. demonstrated that the activation of TLR4 signaling in hepatocytes was imperative, while TLR4 signaling in Kupffer cells was not necessary in diet-induced NAFLD (76). Furthermore, studies based on the Cre-loxP strategy further showed that ablation of TLR4 in hepatocytes led to markedly decreased hepatic inflammation, and improved hepatic steatosis and liver injury (77). In contrast, ablation of TLR4 in myeloid cells did not protect mice from diet-induced hepatic steatosis, and even worsened the systemic inflammatory state (77). Therefore, together with these reports, our data highlight the importance of hepato- 
cytes in response to extracellular stimuli and distinct functions of liver cell populations in the development of chronic liver diseases.

Our in vitro experiments showed that Sparcl1 can bind with TLR4 in hepatocytes, suggesting that Sparcl1 might act as an endogenous ligand of TLR4. Functional studies demonstrated that the inflammatory response evoked by Sparcl1 is dependent on steatosis. Given that acute Sparcl1 treatment can aggravate liver injury and enhance the hepatic inflammatory response in both HFHC diet-fed and HFD-fed mice, but not in the $\mathrm{CCl}_{4}$ treated mice, our results suggest that the role of Sparcl1 in liver metabolism and inflammation might be dependent on a state of metabolic disorders, such as obesity and hyperlipidemia. It has been reported that free fatty acids (FFAs) are not direct TLR agonists and trigger the activation of TLR4 signaling pathway to elicit inflammatory response through indirect mechanisms (78). We found that incubation of palmitate at a dose of $0.2 \mathrm{mM}$ did not induce NF- $\kappa \mathrm{B}$ activation in Hepa1-6 cells. Our data are consistent with 2 previous reports showing that palmitate treatment at a dose of $0.25-1.0 \mathrm{mM}$ cannot activate NF- $\mathrm{BB}$ signaling in human primary adipocytes, 3T3-L1 adipocytes, and mouse primary bone marrow-derived macrophages $(79,80)$. Thus, we propose 2 possibilities regarding the involvement of FFAs and Sparcl1 in TLR4 activation: (a) the Sparcl1/TLR4-mediated hepatic inflammatory response may require the presence of FFAs, and (b) a physical association between FFAs and Sparcl1 might exist.

Mechanistically, we found that Sparcl1 can activate the transcription and expression of several chemokines, including CCL2, Cxcl1, $\mathrm{Cxcl} 2$, and $\mathrm{Cxcl} 5$. Chemokines are chemotactic proteins that act as chemoattractants for leukocyte trafficking, growth, and activation in inflammatory sites (81). In the liver, hepatocytes can produce approximately 50 chemokines, while their receptors are typically expressed in immune cells and hepatic stellate cells (82). Extensive experimental and clinical studies have elucidated the pivotal roles played by the chemokine system in the progression of chronic liver diseases (83). Expression levels of these chemokines are highly upregulated in human NASH, but not in human simple steatosis or HFD-induced fatty liver in mice $(84,85)$. Notably, adenovirus-mediated overexpression of chemokines like Cxcl1 and CCL2 was sufficient to induce NASH phenotypes in obese mice $(85,86)$. Moreover, CCL2 expression in hepatocytes has been shown to be associated with TG content both in a diet-induced mouse NASH model and human NAFLD livers $(86,87)$. Consistently, compared with the patients with simple steatosis, levels of circulating CCL2 were elevated in NASH patients, suggesting that the high CCL2 levels might be of importance for NASH progression (88). In contrast, genetic depletion or pharmacological inhibition of CCL2 in mice ameliorated steatosis progression, alleviated the hepatic inflammatory response, and reduced liver injury $(89,90)$. In our study, the exacerbating effects of Sparcl1 on the hepatic inflammatory response and liver injury were abrogated by either genetic knockdown of CCL2 or pharmacological inhibition of CCR2. Furthermore, suppression of CXCR2 markedly blocked Sparcl1-induced neutrophil infiltration and Ly6G expression. Thus, our results suggest that these chemokines might be the primary targets of Sparcl1 in NASH progression.

There are some limitations of this study that we would like to point out. First, as pointed out by a recent study, presumably healthy volunteers may have markers of NAFLD (91). However, due to ethical requirements, it is not appropriate to perform liver biopsy and histopathological examination in healthy humans. Therefore, a strict ALT threshold was used to ameliorate this problem in our study (91). Second, multicenter clinical studies are needed to further confirm the association of plasma Sparcl1 concentrations and NASH severity. Third, although we have identified TLR4 as a receptor for Sparcl1, which part of Sparcl1 recognizes the TLR4 sequence remains to be determined. Further studies are still needed to answer these questions.

In conclusion, our results support the role of Sparcl1 as a regulator of NASH progression, at least in part, through TLR4/NF-кBdependent activation of CCL2. Our findings provide a mechanism to explain how obesity promotes the development of chronic liver inflammation, injury, and fibrosis. In light of this, therapeutic interventions that suppress Sparcl1 function might represent a promising candidate for treating NASH.

\section{Methods}

Animal experiments. Male C57BL/6J mice aged 6 weeks were purchased from the Shanghai Laboratory Animal Company (SLAC). For NASH diet feeding, C57BL/6J mice were fed a diet containing $40 \%$ fat, $22 \%$ fructose, and 2\% cholesterol (D09100310, Research Diets Inc.) for 12 , 28, or 36 weeks. For HFD feeding, C57BL/6J mice were fed a diet containing $60 \mathrm{kcal} \%$ fat, $20 \mathrm{kcal} \%$ carbohydrate, and $20 \mathrm{kcal} \%$ protein (D12492, Research Diets Inc.) for 12 weeks. The Sparcl1-KO mice were generated by Biocytogen Co. Ltd. using the CRISPR/Cas9-based Extreme Genome Editing system. Two single guide RNAs (sgRNAs) were designed to target upstream of exon 3 and downstream of exon 7 in the nonconserved regions of Sparcl1 using the CRISPR design tool (http://www.sanger.ac.uk/htgt/wge/). The sgRNA plasmids with Cas 9 mRNA were coinjected into C57BL/6J mouse zygotes, and surviving zygotes were transferred into pseudopregnant mice. The genotype of Sparcl1-KO mice was confirmed by PCR amplification and DNA sequencing. Tail tips of each mouse were collected to extract genomic DNA. The targeting region was amplified by PCR (forward 5'-GATTCTGCACCCACTCTGCTCACTT-3', reverse 5'-TCTGGTTGTTGTTGAGCCCCAGAAG-3'). For Sparcl1 treatment, recombinant mouse Sparcl1 protein $(0.2 \mathrm{mg} / \mathrm{kg})$ was intraperitoneally injected into mice. For cenicriviroc treatment, mice were treated daily with cenicriviroc $(30 \mathrm{mg} / \mathrm{kg})$ or vehicle control by intraperitoneal injection. Recombinant adenovirus for Sparcl1 overexpression was generated with GFP adenovirus as the negative control. AAV-delivered short hairpin RNA (shRNA) was constructed using an AAV8 Vector System (GeneChem), driven by CMV or the liver-specific thyroxine-binding globulin (TBG) promoter. For overexpression or knockdown of Sparcl1, $4 \times 10^{9} \mathrm{pfu}$ of purified adenovirus or $5 \times 10^{10} \mathrm{pfu}$ of AAV-shRNA was injected bilaterally into the inguinal fat pads of HFHC diet-fed mice. For knockdown of CCL2, HFHC diet-fed mice were injected with $2 \times$ $10^{9}$ pfu of Ad-shRNA or $2 \times 10^{11}$ pfu of AAV-shRNA by tail vein injection. The 2 shRNAs had the following target sequences: Sparcl1, 5'-GCAAGCAAACCCAAGACTTAA-3'; and CCL2, 5'-GAAGTTGACCCGTAAATCT-3'. For insulin tolerance tests, mice were injected with regular human insulin (Eli Lilly, $0.75 \mathrm{U} / \mathrm{kg}$ ) after a 6-hour fast. Blood glucose was determined using a portable blood glucose meter (LifeScan).

Human studies. A total of 906 adult participants who had undergone liver biopsy were consecutively recruited at the First Affiliated 
Hospital of Wenzhou Medical University from December 2016 to February 2019. Exclusion criteria were as follows: (a) alcohol consumption greater than $140 \mathrm{~g}$ per week in men and greater than $70 \mathrm{~g}$ per week in women; (b) presence of other liver diseases, including hepatitis B or C virus infection, autoimmune hepatitis, primary biliary cholangitis, and liver failure; (c) concomitant use of hepatotoxic drugs, including antibiotics, nonsteroidal antiinflammatory agents, steroids, calcium channel blockers, tamoxifen, amiodarone, isoniazid, and methotrexate; (d) chronic or acute kidney disease as well as presence of urinary tract infection; and (e) fatty liver infiltration less than $5 \%$ on liver histology or missing data. As a result, 689 subjects were excluded and 217 patients with biopsy-confirmed NAFLD were included in the present study. The diagnosis of NAFLD was based on the histopathological features of liver biopsy, including grade of steatosis, hepatocellular ballooning, and lobular inflammation. NAFL (simple steatosis) and NASH were diagnosed by the FLIP algorithm $(39,40)$. Two hundred twenty-eight gender- and age-matched healthy volunteers were enrolled simultaneously according to the following eligibility criteria: (a) BMI less than $25 \mathrm{~kg} / \mathrm{m}^{2}$ and a strict ALT cutoff of $19 \mathrm{U} / \mathrm{L}$ in females and $30 \mathrm{U} / \mathrm{L}$ in males, as described previously (91); (b) no diabetes, no history of cardiovascular disease, and no chronic kidney disease; (c) no clinical evidence of chronic liver diseases, as defined by history, ultrasound features, and biochemical examinations. The baseline characteristics of these individuals are shown in Supplemental Table 2.

ELISA measurement of human Sparcl1. For NAFLD patients, plasma samples were collected on the day of liver biopsy. Gender- and age-matched healthy volunteers were enrolled simultaneously and plasma samples were collected on the same day. All plasma samples were stored in separate tubes at $-80^{\circ} \mathrm{C}$, and kept until ELISA analysis without repeated freezing and thawing. There was no difference in plasma Sparcl1 levels measured at different times in storage (Supplemental Figure 16, A-C). The standard curve of the ELISA assays is shown in Supplemental Figure 16D.

RNA-Seq. High-throughput RNA-Seq was performed by CloudSeq Biotech. Briefly, total RNA was used for removing the rRNAs with the NEBNext rRNA Depletion Kit (New England Biolabs) following the manufacturer's instructions. RNA libraries were constructed by using the NEBNext Ultra II Directional RNA Library Prep Kit (New England Biolabs) according to the manufacturer's instructions. Libraries were controlled for quality and quantified using the BioAnalyzer 2100 system (Agilent Technologies). Library sequencing was performed on an Illumina HiSeq 4000 instrument with 150-bp pairedend reads. Paired-end reads were harvested from the sequencer and quality controlled by Q30. After 3' adaptor trimming and removal of low-quality reads using Cutadapt software (v1.9.3), the high-quality clean reads were aligned to the reference genome (UCSC mm10) with hisat2 software (v2.0.4). Then, guided by the Ensembl gtf gene annotation file, cuffdiff software was used to obtain the gene-level FPKM as the expression profile for mRNA, fold change and $P$ values were calcu- lated based on FPKM, and differentially expressed mRNAs were identified. All original data were deposited in the NCBI's Gene Expression Omnibus database (GEO GSE176681).

Statistics. All statistical analysis was performed with SAS version 9.3 (SAS Institute). Data are presented as mean \pm SEM or mean \pm SD or median (interquartile range). For animal and cellular experiments, a 2-tailed unpaired Student's $t$ test was performed to compare 2 groups. 1-way ANOVA followed by the Student-Newman-Keuls test was used to compare more than 2 groups. For human studies, the correlation of plasma levels of Sparcl1 or CCL2 and clinical parameters was analyzed using Spearman's correlation. The NAFLD patients were classified into different groups according to the FLIP algorithm. A $\chi^{2}$ test was used to compare categorical variables between groups. Receiver operating characteristic (ROC) curve analysis was applied to evaluate the utility of plasma Sparcl1 levels in diagnosing NASH. The differences in diagnostic performance were assessed by DeLong's test. Two-sided $P$ values less than 0.05 were considered statistically significant. Statistical significance is displayed as ${ }^{*} P<0.05,{ }^{* *} P<0.01$, or ${ }^{* * *} P<0.001$.

Study approval. The human study was approved by the Ethics Committee of First Affiliated Hospital of Wenzhou Medical University (Wenzhou, China), in line with the ethical guidelines of the 1975 Declaration of Helsinki. Written informed consent was obtained from each individual. The animal protocol was reviewed and approved by the Animal Care Committees of Zhongshan Hospital, Fudan University (Shanghai, China).

\section{Author contributions}

Y Lu designed and directed the study. BL, LX, JJ, and Wei Liu performed animal and cellular experiments. Wenyue Liu, PZ, YJ, $\mathrm{YH}$, and MZ contributed to the human study. YC, MX, and Y Liu conducted the statistical analysis for the human study. JL and XL contributed to the discussion.

\section{Acknowledgments}

This study was supported by the National Key Research and Development Program of China (no. 2018YFA0800402), the National Natural Science Foundation of China (nos. 81974119, 81722013, and 81970691), and the Original Scientific Research Project of Fudan University (no. IDF152064/002).

Address correspondence to: Yan Lu, Department of Endocrinology and Metabolism, Zhongshan Hospital, Fudan University, 180 Fenglin Road, Xuhui District, Shanghai 200032, China. Phone: 86.21.64041990.693403; Email: lu.yan2@zs-hospital. sh.cn. Or to: Minghua Zheng, MAFLD Research Center, Department of Hepatology, the First Affiliated Hospital of Wenzhou Medical University, 2 Fuxuexiang Road, Wenzhou, Zhejiang Province, China. Phone: 86.577.55578166; Email: zhengmh@ wmu.edu.cn.
1. Malik VS, et al. Nearly a decade on - trends, risk factors and policy implications in global obesity. Nat Rev Endocrinol. 2020;16(11):615-616.

2. Yu Y, et al. Insights into the epidemiology, pathogenesis, and therapeutics of nonalcoholic fatty liver diseases. Adv Sci (Weinh). 2018;6(4):1801585.
3. Friedman SL, et al. Mechanisms of NAFLD development and therapeutic strategies. Nat Med.2018;24(7):908-922.

4. Younossi Z, et al. Global burden of NAFLD and NASH: trends, predictions, risk factors and prevention. Nat Rev Gastroenterol Hepatol. 2018;15(1):11-20.
5. Schuster S, et al. Triggering and resolution of inflammation in NASH. Nat Rev Gastroenterol Hepatol. 2018;15(6):349-364.

6. Kazankov K, et al. The role of macrophages in nonalcoholic fatty liver disease and nonalcoholic steatohepatitis. Nat Rev Gastroenterol Hepatol. 2019;16(3):145-159. 
7. Csak T, et al. Fatty acid and endotoxin activate inflammasomes in mouse hepatocytes that release danger signals to stimulate immune cells. Hepatology. 2011;54(1):133-144.

8. Ekstedt M, et al. Long-term follow-up of patients with NAFLD and elevated liver enzymes. Hepatology. 2006;44(4):865-873.

9. Younossi ZM, et al. Nonalcoholic steatofibrosis independently predicts mortality in nonalcoholic fatty liver disease. Hepatol Commun. 2017;1(5):421-428.

10. Hennessy EJ, et al. Targeting Toll-like receptors: emerging therapeutics? Nat Rev Drug Discov. 2010;9(4):293-307.

11. Bryant CE, et al. The molecular basis of the host response to lipopolysaccharide. Nat Rev Microbiol. 2010;8(1):8-14.

12. Carpino G, et al. Increased liver localization of lipopolysaccharides in human and experimental NAFLD. Hepatology. 2020;72(2):470-485.

13. Kitabatake H, et al. Association between endotoxemia and histological features of nonalcoholic fatty liver disease. World J Gastroenterol. 2017;23(4):712-722

14. du Plessis J, et al. Association of adipose tissue inflammation with histologic severity of nonalcoholic fatty liver disease. Gastroenterology. 2015;149(3):635-648.

15. Azzu V, et al. Adipose tissue-liver cross talk in the control of whole-body metabolism: implications in nonalcoholic fatty liver disease. Gastroenterology. 2020;158(7):1899-1912.

16. Parola M, Marra F. Adipokines and redox signaling: impact on fatty liver disease. Antioxid Redox Signal. 2011;15(2):461-483.

17. Marra F, Bertolani C. Adipokines in liver diseases. Hepatology. 2009;50(3):957-969.

18. Imajo K, et al. Hyperresponsivity to low-dose endotoxin during progression to nonalcoholic steatohepatitis is regulated by leptin-mediated signaling. Cell Metab. 2012;16(1):44-54.

19. Polyzos SA, et al. Circulating leptin in non-alcoholic fatty liver disease: a systematic review and meta-analysis. Diabetologia. 2016;59(1):30-43.

20. Musso G, et al. Adipokines in NASH: postprandial lipid metabolism as a link between adiponectin and liver disease. Hepatology. 2005;42(5):1175-1183.

21. Handa P, et al. Reduced adiponectin signaling due to weight gain results in nonalcoholic steatohepatitis through impaired mitochondrial biogenesis. Hepatology. 2014;60(1):133-145.

22. Guo L, et al. Hepatic neuregulin 4 signaling defines an endocrine checkpoint for steatosis-to-NASH progression. J Clin Invest. 2017;127(12):4449-4461.

23. Klingler A, et al. Species-, organ- and cell-type-dependent expression of SPARCL1 in human and mouse tissues. PLoS One. 2020;15(5):e0233422.

24. Sullivan MM, Sage EH. Hevin/SC1, a matricellular glycoprotein and potential tumor-suppressor of the SPARC/BM-40/Osteonectin family. Int J Biochem Cell Biol. 2004;36(6):991-996.

25. Kos K, Wilding JP. SPARC: a key player in the pathologies associated with obesity and diabetes. Nat Rev Endocrinol. 2010;6(4):225-235.

26. Bai J, et al. Thrombospondin 1 improves hepatic steatosis in diet-induced insulin-resistant mice and is associated with hepatic fat content in humans. EBioMedicine. 2020;57:102849.

27. Icer MA, Gezmen-Karadag M. The multiple functions and mechanisms of osteopontin. Clin Biochem. 2018;59:17-24.

28. Catalán V, et al. Increased tenascin C and Tolllike receptor 4 levels in visceral adipose tissue as a link between inflammation and extracellular matrix remodeling in obesity. J Clin Endocrinol Metab. 2012;97(10):1880-E1889.

29. Clapper JR, et al. Diet-induced mouse model of fatty liver disease and nonalcoholic steatohepatitis reflecting clinical disease progression and methods of assessment. Am J Physiol Gastrointest Liver Physiol. 2013;305(7):483-G495.

30. Zhou B, et al. $\mathrm{N}^{6}$-methyladenosine reader protein YT521-B homology domain-containing 2 suppresses liver steatosis by regulation of mRNA stability of lipogenic genes. Hepatology. 2021;73(1):91-103.

31. Lu Y, et al. Yin Yang 1 promotes hepatic steatosis through repression of farnesoid $\mathrm{X}$ receptor in obese mice. Gut. 2014;63(1):170-178.

32. Bijnen M, et al. Adipose tissue macrophages induce hepatic neutrophil recruitment and macrophage accumulation in mice. Gut. 2018;67(7):1317-1327.

33. Stojsavljević S, et al. Adipokines and proinflammatory cytokines, the key mediators in the pathogenesis of nonalcoholic fatty liver disease. World J Gastroenterol. 2014;20(48):18070-18091.

34. Reggio S, et al. Increased basement membrane components in adipose tissue during obesity: links with TGF $\beta$ and metabolic phenotypes. J Clin Endocrinol Metab. 2016;101(6):2578-2587.

35 . Kos K, et al. Regulation of the fibrosis and angiogenesis promoter SPARC/osteonectin in human adipose tissue by weight change, leptin, insulin, and glucose. Diabetes. 2009;58(8):1780-1788.

36. Mazzolini G, et al. SPARC expression is associated with hepatic injury in rodents and humans with non-alcoholic fatty liver disease. Sci Rep. 2018;8(1):725.

37. Kleiner DE, et al. Design and validation of a histological scoring system for nonalcoholic fatty liver disease. Hepatology. 2005;41(6):1313-1321.

38. Rastogi A, et al. Non-alcoholic fatty liver disease - histological scoring systems: a large cohort single-center, evaluation study. APMIS 2017;125(11):962-973.

39. Bedossa P, FLIP Pathology Consortium. Utility and appropriateness of the fatty liver inhibition of progression (FLIP) algorithm and steatosis, activity, and fibrosis (SAF) score in the evaluation of biopsies of nonalcoholic fatty liver disease. Hepatology. 2014;60(2):565-575

40. Nascimbeni F, et al. Clinical validation of the FLIP algorithm and the SAF score in patients with non-alcoholic fatty liver disease. J Hepatol. 2020;72(5):828-838.

41. Li W, et al. LPS induces active HMGB1 release from hepatocytes into exosomes through the coordinated activities of TLR4 and Caspase-11/ GSDMD Signaling. Front Immunol. 2020;11:229.

42. $\mathrm{MaX}$, et al. Celastrol protects against obesity and metabolic dysfunction through activation of a HSF1-PGC1 $\alpha$ transcriptional axis. Cell Metab.
2015;22(4):695-708.

43. Liu Y, et al. Protein inhibitor of activated STAT 1 (PIAS1) protects against obesity-induced insulin resistance by inhibiting inflammation cascade in adipose tissue. Diabetes. 2015;64(12):4061-4074.

44. Ma X, et al. Role of forkhead box protein A3 in age-associated metabolic decline. Proc Natl Acad Sci U S A. 2014;111(39):14289-14294.

45. Zimmermann HW, Tacke F. Modification of chemokine pathways and immune cell infiltration as a novel therapeutic approach in liver inflammation and fibrosis. Inflamm Allergy Drug Targets. 2011;10(6):509-536.

46. Marra F, Tacke F. Roles for chemokines in liver disease. Gastroenterology. 2014;147(3):577-594.

47. Lee PY, et al. Ly6 family proteins in neutrophil biology. JLeukoc Biol. 2013;94(4):585-594.

48. Ye D, et al. Lipocalin-2 mediates non-alcoholic steatohepatitis by promoting neutrophil-macrophage crosstalk via the induction of CXCR2 J Hepatol. 2016;65(5):988-997.

49. Tacke F. Cenicriviroc for the treatment of nonalcoholic steatohepatitis and liver fibrosis. Expert Opin Investig Drugs. 2018;27(3):301-311.

50. Ratziu V, et al. Cenicriviroc treatment for adults with nonalcoholic steatohepatitis and fibrosis: final analysis of the phase $2 \mathrm{~b}$ CENTAUR study. Hepatology. 2020;72(3):892-905.

51. Mora E, et al. Bindarit: an anti-inflammatory small molecule that modulates the NFאB pathway. Cell Cycle. 2012;11(1):159-169.

52. Huang L, et al. Transcription factor Hlx controls a systematic switch from white to brown fat through Prdm16-mediated co-activation. Nat Commun. 2017;8(1):68.

53. Chen Q, et al. Cbx 4 sumoylates Prdm16 to regulate adipose tissue thermogenesis. Cell Rep. 2018;22(11):2860-2872.

54. Bates R, et al. Adipose tissue: an emerging target for adeno-associated viral vectors. Mol Ther Methods Clin Dev. 2020;19:236-249.

55. Chen S, et al. The phytochemical hyperforin triggers thermogenesis in adipose tissue via a Dlat-AMPK signaling axis to curb obesity. Cell Metab. 2021;33(3):565-580.

56. Tan-Garcia A, et al. Liver fibrosis and CD206 ${ }^{+}$macrophage accumulation are suppressed by anti-GM-CSF therapy. JHEP Rep. 2019;2(1):100062.

57. Guo Q, et al. Integrin $\beta_{1}$-enriched extracellular vesicles mediate monocyte adhesion and promote liver inflammation in murine NASH. J Hepatol. 2019;71(6):1193-1205.

58. Widjaja AA, et al. Inhibiting interleukin 11 signaling reduces hepatocyte death and liver fibrosis, inflammation, and steatosis in mouse models of nonalcoholic steatohepatitis. Gastroenterology. 2019;157(3):777-792.

59. Cai D, et al. Local and systemic insulin resistance resulting from hepatic activation of IKK-beta and NF-kappaB. Nat Med. 2005;11(2):183-190.

60. Okin D, Medzhitov R. The effect of sustained inflammation on hepatic mevalonate pathway results in hyperglycemia. Cell. 2016;165(2):343-356.

61. Eslam M, et al. MAFLD: a consensus-driven proposed nomenclature for metabolic associated fatty liver disease. Gastroenterology. 
2020;158(7):1999-2014.

62. Tilg H, Moschen AR. Evolution of inflammation in nonalcoholic fatty liver disease: the multiple parallel hits hypothesis. Hepatology. 2010;52(5):1836-1846.

63. Zhao GN, et al. Tmbim1 is a multivesicular body regulator that protects against non-alcoholic fatty liver disease in mice and monkeys by targeting the lysosomal degradation of Tlr4. Nat Med. 2017;23(6):742-752.

64. Stienstra R, et al. Kupffer cells promote hepatic steatosis via interleukin-1beta-dependent suppression of peroxisome proliferator-activated receptor alpha activity. Hepatology. 2010;51(2):511-522.

65. Day CP, James OF. Steatohepatitis: a tale of two "hits"? Gastroenterology. 1998;114(4):842-845.

66. Wu M, et al. Cbf $\beta$ governs osteoblast-adipocyte lineage commitment through enhancing $\beta$-catenin signaling and suppressing adipogenesis gene expression. Proc Natl Acad Sci U S A. 2017;114(38):10119-10124.

67. Wu Z, et al. CD146 is a novel ANGPTL2 receptor that promotes obesity by manipulating lipid metabolism and energy expenditure. $\mathrm{Adv} S \mathrm{Sci}$ (Weinh). 2021;8(6):2004032.

68. $\mathrm{Hu} \mathrm{X}$, et al. Brd4 modulates diet-induced obesity via PPAR $\gamma$-dependent Gdf3 expression in adipose tissue macrophages. JCI Insight. 2021;6(7):e143379.

69. Yang Y, et al. Cysteine-rich protein 61 regulates adipocyte differentiation from mesenchymal stem cells through mammalian target of rapamycin complex 1 and canonical Wnt signaling. FASEB J. 2018;32(6):3096-3107.

70. Fischer C, et al. A miR-327-FGF10-FGFR2-mediated autocrine signaling mechanism controls white fat browning. Nat Commun. 2017;8(1):2079.

71. Chen Y, et al. Adipose vascular endothelial growth factor B is a major regulator of energy metabolism. JEndocrinol. 2020;244(3):511-521.

72. Tsuchida T, et al. A simple diet- and chemicalinduced murine NASH model with rapid progression of steatohepatitis, fibrosis and liver cancer. J Hepatol. 2018;69(2):385-395.

73. Blanc V, et al. Apobec1 complementation factor overexpression promotes hepatic steatosis, fibrosis, and hepatocellular cancer. J Clin Invest. 2021;131(1):e138699.

74. Sharifnia T, et al. Hepatic TLR4 signaling in obese NAFLD. Am J Physiol Gastrointest Liver Physiol. 2015;309(4):270-G278.

75. Ye D, et al. Toll-like receptor-4 mediates obesityinduced non-alcoholic steatohepatitis through activation of X-box binding protein-1 in mice. Gut. 2012;61(7):1058-1067.

76. Li L, et al. Nuclear factor high-mobility group box1 mediating the activation of Toll-like receptor 4 signaling in hepatocytes in the early stage of nonalcoholic fatty liver disease in mice. Hepatology. 2011;54(5):1620-1630.

77. Jia L, et al. Hepatocyte Toll-like receptor 4 regulates obesity-induced inflammation and insulin resistance. Nat Commun. 2014;5:3878.

78. Korbecki J, Bajdak-Rusinek K. The effect of palmitic acid on inflammatory response in macrophages: an overview of molecular mechanisms. Inflamm Res. 2019;68(11):915-932.

79. Pal D, et al. Fetuin-A acts as an endogenous ligand of TLR4 to promote lipid-induced insulin resistance. Nat Med. 2012;18(8):1279-1285.

80. Lancaster GI, et al. Evidence that TLR4 is not a receptor for saturated fatty acids but mediates lipid-induced inflammation by reprogramming macrophage metabolism. Cell Metab. 2018;27(5):1096-1110.

81. Sahin H, et ak. Functional role of chemokines in liver disease models. Nat Rev Gastroenterol Hepatol. 2010;7(12):682-690.

82. Roh YS, Seki E. Chemokines and chemokine receptors in the development of NAFLD. Adv Exp Med Biol. 2018;1061:45-53.

83. Chen W, et al. Function and therapeutic advances of chemokine and its receptor in nonalcoholic fatty liver disease. Therap Adv Gastroenterol. 2018;11:1756284818815184.

84. Bertola A, et al. Hepatic expression patterns of inflammatory and immune response genes associated with obesity and NASH in morbidly obese patients. PLoS One. 2010;5(10):e13577.

85. Hwang S, et al. Interleukin-22 Ameliorates neutrophil-driven nonalcoholic steatohepatitis through multiple targets. Hepatology. 2020;72(2):412-429.

86. Obstfeld AE, et al. C-C chemokine receptor 2 (CCR2) regulates the hepatic recruitment of myeloid cells that promote obesity-induced hepatic steatosis. Diabetes. 2010;59(4):916-925.

87. Westerbacka J, et al. Genes involved in fatty acid partitioning and binding, lipolysis, monocyte/macrophage recruitment, and inflammation are overexpressed in the human fatty liver of insulin-resistant subjects. Diabetes. 2007;56(11):2759-2765.

88. Haukeland JW, et al. Systemic inflammation in nonalcoholic fatty liver disease is characterized by elevated levels of CCL2. J Hepatol. 2006;44(6):1167-1174.

89. Kanda H, et al. MCP-1 contributes to macrophage infiltration into adipose tissue, insulin resistance, and hepatic steatosis in obesity. J Clin Invest. 2006;116(6):1494-1505.

90. Baeck C, et al. Pharmacological inhibition of the chemokine CCL2 (MCP-1) diminishes liver macrophage infiltration and steatohepatitis in chronic hepatic injury. Gut. 2012;61(3):416-426.

91. Takyar V, et al. How healthy are the "Healthy volunteers"? Penetrance of NAFLD in the biomedical research volunteer pool. Hepatology. 2017;66 (3):825-833. 